ALEA, Lat. Am. J. Probab. Math. Stat. 18, 793-828 (2021)

DOI: $10.30757 /$ ALEA.v18-29

\title{
Limit theorems for $U$-statistics of Bernoulli data
}

\section{Davide Giraudo}

Ruhr-Universität Bochum

Universitätsstr. 150,

Bochum, Germany.

E-mail address: davide.giraudo@rub.de

URL: https://sites.google.com/site/davidegiraudomathematics/home

\begin{abstract}
In this paper, we consider $U$-statistics whose data is a strictly stationary sequence which can be expressed as a functional of an i.i.d. one. We establish a strong law of large numbers, a bounded law of the iterated logarithms and a central limit theorem under a dependence condition. The main ingredients for the proof are an approximation by $U$-statistics whose data is a functional of $\ell$ i.i.d. random variables and an analogue of the Hoeffding's decomposition for $U$-statistics of this type.
\end{abstract}

\section{Introduction and main results}

1.1. Context. Let $\left(X_{j}\right)_{j \geq 1}$ be a strictly stationary sequence, in the sense that the vectors $\left(X_{i}\right)_{i=1}^{n}$ and $\left(X_{i+k}\right)_{i=1}^{n}$ have the same distribution for all $n$ and $k \geq 1$. The $U$-statistic of kernel $h: \mathbb{R} \times \mathbb{R} \rightarrow \mathbb{R}$ and data $\left(X_{j}\right)_{j \geq 1}$ is defined as

$$
U_{n}:=\sum_{1 \leq i<j \leq n} h\left(X_{i}, X_{j}\right), \quad n \geq 2 .
$$

The study of the asymptotic behavior of the sequence $\left(U_{n}\right)_{n>2}$ properly normalized is a question of interest in probability theory and the applications. We will be interested in the following three limit theorems.

(1) Law of large numbers: let $1 \leq p<2$; the following convergence holds

$$
\frac{1}{n^{1+1 / p}}\left(U_{n}-\mathbb{E}\left[U_{n}\right]\right) \rightarrow 0 \text { a. s.. }
$$

Received by the editors April 12th, 2020; accepted February 9th, 2021.

2010 Mathematics Subject Classification. 60F05, 60F15, 60G99.

Key words and phrases. Law of large numbers, law of the iterated logarithms, central limit theorem, $U$-statistics, functionals of i.i.d.

Research supported by the German National Academic Foundation and Collaborative Research Center SFB 823 "Statistical modelling of nonlinear dynamic processes". 
(2) Bounded law of the iterated logarithms: the random variable

$$
\sup _{n \geq 1} \frac{1}{n^{3 / 2} \sqrt{\operatorname{LL}(n)}}\left|U_{n}-\mathbb{E}\left[U_{n}\right]\right|
$$

is almost surely finite, where $L: \mathbb{R}_{+} \rightarrow \mathbb{R}_{+}$is defined by $L(x)=$ $\max \{\ln x, 1\}$ and $\operatorname{LL}(x):=L \circ L(x)$.

(3) Central limit theorem: there exists a $\sigma>0$ such that

$$
\frac{1}{n^{3 / 2}}\left(U_{n}-\mathbb{E}\left[U_{n}\right]\right) \rightarrow \sigma N \text { in distribution, }
$$

where $N$ is a standard normal random variable.

Usually, the conditions for guarantying this kind of limit theorems are on the dependence of the sequence $\left(X_{j}\right)_{j \geq 1}$ and also on the kernel $h$, for example by requiring some integrability conditions on $h\left(X_{1}, X_{2}\right)$. We will first review a few results for the case where the data $\left(X_{j}\right)_{j \geq 1}$ is i.i.d. and the kernel $h$ is symmetric.

(1) If $1 \leq p<2$ and $h\left(X_{1}, X_{2}\right) \in \mathbb{L}^{p}$, then (1.2) holds Giné and Zinn (1992).

(2) If $h\left(X_{1}, X_{2}\right) \in \mathbb{L}^{2}$, then the random variable defined by (1.3) is almost surely finite. Moreover, for all $1<p<2$, according to Theorem 2.5 in Arcones and Giné (1995), the following inequality holds:

$\left\|\sup _{n \geq 1} \frac{1}{\sqrt{n L(L(n))}}\left|U_{n}\left(h, f,\left(\varepsilon_{i}\right)_{i \in \mathbb{Z}}\right)-\mathbb{E}\left[U_{n}\left(h, f,\left(\varepsilon_{i}\right)_{i \in \mathbb{Z}}\right)\right]\right|\right\|_{p} \leq C_{p}\left\|h\left(X_{1}, X_{2}\right)\right\|_{2}$.

(3) The convergence (1.4) has been established in Hoeffding (1948).

The extension of these results to the case of stationary dependent data is a challenging problem. The law of large numbers has been established under a $\beta$ mixing assumption in Arcones (1998a); Dehling and Sharipov (2009). In the case $p=1$, the independence assumption can be replaced by a 4-dependence assumption, that is, for all 4 -uple of distinct integers $\left(i_{1}, i_{2}, i_{3}, i_{4}\right)$, the collection of random variables $\left(X_{i_{k}}\right)_{k=1}^{4}$ is independent. If the sequence $\left(X_{j}\right)_{j \geq 1}$ is identically distributed, then the law of large numbers hold (Theorem 1 in Arcones (1998b)). The question of an equivalent of the ergodic theorem when the data is any strictly stationary sequence was considered in Aaronson et al. (1996). The question of the law of the iterated logarithms was also adressed in Dehling and Wendler (2010b) where the data is allowed to be $\alpha$-mixing or a functional of a $\beta$-mixing sequence. A central limit theorem has been established under a $\beta$-mixing assumption in Arcones (1995) and under an $\alpha$-mixing condition in Dehling and Wendler (2010a). The $\tau$-dependent case has been addressed in Leucht (2012). The case of associated random variables has also been investigated in Dewan and Prakasa Rao (2002). Note that there are also results dealing with the asymptotic behavior of $U$-statistics of a semimartingale (Podolskij et al., 2014) and of Poisson processes (Reitzner and Schulte, 2013).

In this paper, we will be interested in establishing the law of large number, the law of the iterated logarithms and the central limit theorem when the data can be expressed as a functional of an i.i.d. sequence. In a similar context on the data but for weighted $U$-statistics, the central limit theorem was investigated in Hsing and Wu (2004).

Let us precise the context. Let $\left(\varepsilon_{i}\right)_{i \in \mathbb{Z}}$ be an independent identically distributed sequence with values in $\mathbb{R}, k \geq 1$. Given measurable functions $h: \mathbb{R} \times \mathbb{R} \rightarrow \mathbb{R}$ and 
$f: \mathbb{R}^{\mathbb{Z}} \rightarrow \mathbb{R}$, we are interested in the asymptotic behavior of the $U$-statistic of order two defined by

$$
U_{n}\left(h, f,\left(\varepsilon_{i}\right)_{i \in \mathbb{Z}}\right)=\sum_{1 \leq i<j \leq n} h\left(f\left(\left(\varepsilon_{i-k}\right)_{k \in \mathbb{Z}}\right), f\left(\left(\varepsilon_{j-k}\right)_{k \in \mathbb{Z}}\right)\right),
$$

that is, letting $X_{j}:=f\left(\left(\varepsilon_{j-k}\right)_{k \in \mathbb{Z}}\right), U_{n}$ is a $U$-statistic of kernel $h$ and the data is the strictly stationary sequence $\left(X_{j}\right)_{j \geqslant 1}$. More precisely, we are interested in conditions involving the kernel $h$ and the sequence $\left(f\left(\left(\varepsilon_{j-k}\right)_{k \in \mathbb{Z}}\right)\right)_{j \geq 1}$ which guarantee the previously mentioned limit theorems. Sequences of the aforementioned form are among the most natural examples of strictly stationary processes. Indeed, they cover for instance the case of Hölderian functions of linear processes, function of linear processes with independent Gaussian innovation (in connection with chaos expansion) and Volterra series, that is, processes of the form $X_{j}=\sum_{i_{1}<i_{2}} a_{i_{1}, i_{2}} \varepsilon_{j-i_{1}} \varepsilon_{j-i_{2}}$. The central limit theorem was considered (Hsing and $\mathrm{Wu}, 2004$ ) and a law of large numbers was established in Borovkova et al. (2001). We aim to complement the results in this area by giving rates in the law of large numbers for $U$-statistics whose data is a function of an i.i.d. sequence and also provide a general tool for dealing with this type of $U$-statistics, namely, a generalized Hoeffding's decomposition. We also study the bounded law of the iterated logarithms via the control of a maximal function, which could be of use in order to refine the consistency that we could derive with an application of the law of large numbers. It is also an interesting theoretical challenge to have checkable sufficient conditions for boundedness with respect to $n$ of normalized $U$-statistics with the weakest possible normalization.

In all the paper, the kernel $h$ is supposed to be symmetric in the sense that $h(x, y)=h(y, x)$ for all $x, y \in \mathbb{R}$.

The paper is organized as follows. In Subsection 1.2, we will introduce a measure of dependence of a $U$-statistic whose data is a functional of an i.i.d. sequence. In Subsection 1.3, we formulate an analogue of the Hoeffding decomposition for such $U$-statistics. Subsections 1.4, 1.5 and 1.6 are devoted respectively to the statements of the law of large numbers, the bounded law of the iterated logarithms and the central limit theorems for $U$-statistics of Bernoulli data. In Subsection 1.7, we give examples of kernels $h$ for which the measure of dependence can be estimated with the help of the dependence of the data. Section 2 is devoted to the proofs of the previously mentioned results.

1.2. Measure of dependence. The extension of the results of the i.i.d. case requires a measure of dependence. Let $\left(\varepsilon_{u}\right)_{u \in \mathbb{Z}}$ be an i.i.d. sequence, $f: \mathbb{R}^{\mathbb{Z}} \rightarrow \mathbb{R}$ a measurable function and $h: \mathbb{R} \times \mathbb{R} \rightarrow \mathbb{R}$. In order to deal with the dependence which comes into play in $U_{n}\left(h, f,\left(\varepsilon_{i}\right)_{i \in \mathbb{Z}}\right)$, we need the following notations. Denote $X_{j}:=$ $f\left(\left(\varepsilon_{j-i}\right)_{i \in \mathbb{Z}}\right)$ and define the random vectors

$$
V_{j, \ell}:=\left(\varepsilon_{u}\right)_{u=j-\ell}^{j+\ell} .
$$

The random variable $\mathbb{E}\left[X_{j} \mid V_{j, \ell}\right]$ can be writen as a function of $V_{j, \ell}$ and by stationarity and Lemma A.6, the involved function does not depend on $j$. Therefore, we write

$$
\mathbb{E}\left[X_{j} \mid V_{j, \ell}\right]=f_{\ell}\left(V_{j, \ell}\right)
$$


We then define for $p \geq 1$ and $\ell \geq 1$ the coefficient of dependence

$$
\theta_{\ell, p}:=\sup _{j \geq 0}\left\|h\left(f_{\ell}\left(V_{0, \ell}\right), f_{\ell}\left(V_{j, \ell}\right)\right)-h\left(f_{\ell-1}\left(V_{0, \ell-1}\right), f_{\ell-1}\left(V_{j, \ell-1}\right)\right)\right\|_{p}
$$

and for $\ell=0, \theta_{0, p}=\sup _{j \geq 0}\left\|h\left(f_{0}\left(V_{j, \ell}\right), f_{0}\left(V_{0, \ell}\right)\right)\right\|_{p}$.

In particular, finiteness of $\sum_{\ell \geq 1} \theta_{\ell, p}$ allows to write

$$
\begin{aligned}
& U_{n}\left(h, f,\left(\varepsilon_{i}\right)_{i \in \mathbb{Z}}\right)=\sum_{1 \leq i<j \leq n} h\left(f_{0}\left(V_{i, 0}\right), f_{0}\left(V_{j, 0}\right)\right) \\
& \quad+\sum_{\ell \geq 1} \sum_{1 \leq i<j \leq n} h\left(f_{\ell}\left(V_{i, \ell}\right), f_{\ell}\left(V_{j, \ell}\right)\right)-h\left(f_{\ell-1}\left(V_{i, \ell-1}\right), f_{\ell-1}\left(V_{j, \ell-1}\right)\right),
\end{aligned}
$$

where the convergence takes place in $\mathbb{L}^{p}$ and almost surely. The interest of the decomposition (1.9) is that it reduces the treatmeant of the original $U$-statistic to that of $U$-statistics whose data is a strictly stationnary sequence which is a functional of $2 \ell+1$ independent identically distributed random variables. Nevertheless, this task requires some work in order to be reduced to $U$-statistics of independent data.

Note that in the particular case where $h(x, y)=x+y$, the coefficient $\theta_{\ell, p}$ can be estimated (see Theorem 1 in $\mathrm{Wu}, 2005$ )by the inequality

$$
\theta_{\ell, p} \leq 2\left\|f_{\ell}\left(V_{0, \ell}\right)-f_{\ell-1}\left(V_{0, \ell-1}\right)\right\|_{p}
$$

which can be bounded in turn by $2 \delta_{\ell, p}+2 \delta_{-\ell, p}$, where for $k \in \mathbb{Z}$,

$$
\delta_{k, p}=\left\|X_{k}-X_{k}^{*}\right\|_{p}
$$

$X_{k}^{*}=f\left(\left(\varepsilon_{k-u}^{*}\right)_{u \in \mathbb{Z}}\right), \varepsilon_{u}^{*}=\varepsilon_{u}$ if $u \neq 0$ and $\varepsilon_{0}^{*}=\varepsilon_{0}^{\prime}$, where $\varepsilon_{0}^{\prime}$ has the same law as $\varepsilon_{0}$ and is independent of $\left(\varepsilon_{u}\right)_{u \in \mathbb{Z}}$.

In some other cases, the dependence coefficient $\theta_{\ell, p}$ can be bounded in terms of $\delta_{k, p}$; see Subsection 1.7 for more details.

1.3. A generalized Hoeffding's decomposition. A usefull tool to establish limit theorems for $U$-statistics with i.i.d. data is the Hoeffdings's decomposition (Hoeffding, 1948). Let $h: \mathbb{R}^{k} \times \mathbb{R}^{k} \rightarrow \mathbb{R}$ be a symmetric measurable function and $\left(X_{j}\right)_{j \in \mathbb{Z}}$ be an i.i.d. sequence. We write decompose $h$ in the following way:

$$
h(x, y)=\theta+h_{1}(x)+h_{1}(y)+h_{2}(x, y),
$$

where $\theta=\mathbb{E}\left[h\left(X_{1}, X_{2}\right)\right]$,

$$
\begin{gathered}
h_{1}(x)=\mathbb{E}\left[h\left(X_{1}, x\right)\right]-\theta \text { and } \\
h_{2}(x, y)=h(x, y)-h_{1}(x)-h_{1}(y)-\theta .
\end{gathered}
$$

In this way, the following equality holds

$$
\sum_{1 \leq i<j \leq n} h\left(X_{i}, X_{j}\right)=\left(\begin{array}{l}
n \\
2
\end{array}\right) \theta+n \sum_{i=1}^{n} h_{1}\left(X_{i}\right)+\sum_{1 \leq i<j \leq n} h_{2}\left(X_{i}, X_{j}\right) .
$$

The part involving $h_{2}$ can be treated by martingale techniques, since the sequence $\left(\sum_{i=1}^{j-1} h_{2}\left(X_{i}, X_{j}\right)\right)_{j \geq 1}$ is a martingale differences sequence with respect to the filtration $\left(\sigma\left(X_{u}, u \leq j\right)\right)$ and the terms $\sum_{i=1}^{j-1} h_{2}\left(X_{i}, X_{j}\right)$ can be treated thanks to a reverse martingale differences property. 
We would like to extend this to the setting mentioned in 1.1, that is, $X_{j}:=$ $f\left(\left(\varepsilon_{j-k}\right)_{k \in \mathbb{Z}}\right)$, where $\left(\varepsilon_{u}\right)_{u \in \mathbb{Z}}$ is an i.i.d. sequence. We introduce the following notation

$$
U_{n}^{\text {ind }}\left(h,\left(\varepsilon_{i}\right)_{i \in \mathbb{Z}}\right)=\sum_{1 \leq i<j \leq n} h\left(\varepsilon_{i}, \varepsilon_{j}\right),
$$

where $\left(\varepsilon_{i}\right)_{i \in \mathbb{Z}}$ is an i.i.d. sequence of random variables with values in $\mathbb{R}^{k}$ and $h: \mathbb{R}^{k} \times \mathbb{R}^{k} \rightarrow \mathbb{R}$ is a measurable function.

One naturally expects the decomposition to be more complicated than in the independent case. Let us point out the major differences and common points. Like in the independent case, the decomposition of the centered $U$-statistic involves a stationary sequence and a degenerated $U$-statistic. But in the context of Bernoulli data, one gets a series involving stationary sequences and a series of degenerated $U$-statistics. Another difference is that we also have remainder terms which are not directly associated to the involved stationary sequences or degenerated $U$-statistic. The origin of these terms will be explained during the proof.

Proposition 1.1. Let $\left(\varepsilon_{u}\right)_{u \in \mathbb{Z}}$ be an i.i.d. sequence of random variables, $\left(\varepsilon_{u}^{\prime}\right)_{u \in \mathbb{Z}}$ an independent copy of $\left(\varepsilon_{u}\right)_{u \in \mathbb{Z}}$ and $f: \mathbb{R}^{\mathbb{Z}} \rightarrow \mathbb{R}$ be a measurable function. Let $V_{k, \ell}:=\left(\varepsilon_{u}\right)_{u=k-\ell}^{k+\ell}$ and $V_{k, \ell}^{\prime}:=\left(\varepsilon_{u}^{\prime}\right)_{u=k-\ell}^{k+\ell}$. Let $f_{\ell}: \mathbb{R}^{2 \ell+1} \rightarrow \mathbb{R}$ be a function such that

$$
f_{\ell}\left(V_{k, \ell}\right)=\mathbb{E}\left[f\left(\left(\varepsilon_{k-u}\right)_{u \in \mathbb{Z}}\right) \mid V_{k, \ell}\right] \text { a.s. }
$$

Let $h: \mathbb{R} \times \mathbb{R} \rightarrow \mathbb{R}$ be a symmetric measurable function. Assume that the following convergence holds almost surely for all $1 \leq i<j$ :

$$
\lim _{\ell \rightarrow+\infty} h\left(f_{\ell}\left(V_{i, \ell}\right), f_{\ell}\left(V_{j, \ell}\right)\right)=h\left(f\left(\left(\varepsilon_{i-u}\right)_{u \in \mathbb{Z}}\right), f\left(\left(\varepsilon_{j-u}\right)_{u \in \mathbb{Z}}\right)\right) .
$$

Then the following equality holds:

$$
\begin{aligned}
U_{n}\left(h, f,\left(\varepsilon_{i}\right)_{i \in \mathbb{Z}}\right)-\mathbb{E}\left[U_{n}\left(h, f,\left(\varepsilon_{i}\right)_{i \in \mathbb{Z}}\right)\right]=n \sum_{k=1}^{n} \mathbb{E}\left[h\left(f_{0}\left(V_{k, 0}\right), f_{0}\left(V_{k, 0}^{\prime}\right)\right) \mid V_{k, 0}\right] \\
\quad+\sum_{\ell \geq 1}(4 \ell+1)\left\lfloor\frac{n}{4 \ell+1}\right\rfloor \sum_{k=1}^{(4 \ell+2)\left\lfloor\frac{n}{4 \ell+2}\right\rfloor+1} Y_{k}^{(\ell)}+U_{n}^{\text {ind }}\left(h^{(0)},\left(\varepsilon_{i}\right)_{i}\right) \\
\quad+\sum_{\ell \geq 1} \sum_{a, b \in[4 \ell+2]} U_{\left\lfloor\frac{n}{2 \ell}\right\rfloor}^{\text {ind }}\left(h_{a, b}^{(\ell)},\left(\varepsilon_{i}^{a, b}\right)\right)+R_{n, 1,1}+R_{n, 1,2}+\sum_{k=2}^{6} R_{n, k}, \quad(1.19)
\end{aligned}
$$

where for each $\ell \geq 1$ and all $a, b \in[4 \ell+2]$, the $U$-statistic $U_{\left\lfloor\frac{n}{2 \ell}\right\rfloor}^{\text {ind }}\left(h_{a, b}^{(\ell)},\left(\varepsilon_{i}^{a, b}\right)\right)$ has independent data and is degenerated, the random variable $Y_{k}^{(\ell)}$ is defined by $Y_{k}^{(\ell)}=\mathbb{E}\left[h\left(f_{\ell}\left(V_{k, \ell}\right), f_{\ell}\left(V_{0, \ell}^{\prime}\right)\right) \mid V_{k, \ell}\right]-\mathbb{E}\left[h\left(f_{\ell-1}\left(V_{k, \ell-1}\right), f_{\ell-1}\left(V_{0, \ell-1}^{\prime}\right)\right) \mid V_{k, \ell-1}\right]$, the function $h_{a, b}^{(\ell)}: \mathbb{R}^{4 \ell+2} \times \mathbb{R}^{4 \ell+2} \rightarrow \mathbb{R}$ is defined by

$$
\begin{aligned}
h_{a, b}^{(\ell)}:=h_{a, b} & \left(\left(x_{i}\right)_{i=1}^{4 \ell+2},\left(y_{j}\right)_{j=1}^{4 \ell+2}\right) \\
& \quad-h_{a, b}\left(\left(x_{i}\right)_{i=1}^{4 \ell+2}, \varepsilon_{0}^{a, b}\right)-h_{a, b}\left(\left(y_{j}\right)_{j=1}^{4 \ell+2}, \varepsilon_{0}^{a, b}\right)+\mathbb{E}\left[h_{a, b}\left(\varepsilon_{0}^{a, b}, \varepsilon_{0}^{\prime a, b}\right)\right]
\end{aligned}
$$

and the function $h_{a, b}: \mathbb{R}^{4 \ell+2} \times \mathbb{R}^{4 \ell+2} \rightarrow \mathbb{R}$ is defined for $0 \leq a-b \leq 2 \ell$ by 


$$
\begin{aligned}
& h_{a, b}\left(\left(x_{i}\right)_{i=1}^{4 \ell+2},\left(y_{j}\right)_{j=1}^{4 \ell+2}\right):=h\left(\left(x_{i+a-b}\right)_{i=1}^{2 \ell+1},\left(y_{j}\right)_{j=1}^{2 \ell+1}\right)+h\left(\left(y_{j+a-b}\right)_{j=1}^{2 \ell+1},\left(x_{i}\right)_{i=1}^{2 \ell+1}\right) \\
& \text { and for }(2 \ell+1) \leq a-b \leq(4 \ell+2)-1 \text { by } \\
& \qquad \begin{array}{l}
h_{a, b}\left(\left(x_{i}\right)_{i=1}^{4 \ell+2},\left(y_{j}\right)_{j=1}^{4 \ell+2}\right) \\
\quad:=h\left(\left(x_{i+a-b-(2 \ell+1)}\right)_{i=1}^{2 \ell+1},\left(y_{j}\right)_{j=1}^{2 \ell+1}\right)+h\left(\left(y_{j+a-b-(2 \ell+1)}\right)_{j=1}^{2 \ell+1},\left(x_{i}\right)_{i=1}^{2 \ell+1}\right),
\end{array}
\end{aligned}
$$

the random vectors $\varepsilon_{u}^{a, b}$ are defined by

$$
\begin{gathered}
\varepsilon_{u}^{a, b}:=\left(\varepsilon_{j}\right)_{j=u(4 \ell+2)+b-\ell}^{(u+1)(4 \ell+2)+b-\ell-1}, 0 \leq a-b \leq 2 \ell ; \\
\varepsilon_{u}^{a, b}:=\left(\varepsilon_{j}\right)_{j=u(4 \ell+2)+b+\ell+1}^{(u+1)(4 \ell+2)+b+\ell},(2 \ell+1) \leq a-b \leq(4 \ell+2)-1 ;
\end{gathered}
$$

and the remainder terms are defined as

$$
\begin{aligned}
& R_{n, 1,1}:=\sum_{\ell \geq 1} \sum_{j=(4 \ell+2)\left\lfloor\frac{n}{4 \ell+2}\right\rfloor+1}^{n} \sum_{i=(4 \ell+2)\left\lfloor\frac{j-1}{4 \ell+2}\right\rfloor+1}^{j-1} H_{i, j}^{(\ell)} \\
& R_{n, 1,2}:=\sum_{\ell \geq 1} \sum_{a=1}^{4 \ell+2} \sum_{j=(4 \ell+2)\left\lfloor\frac{n}{4 \ell+2}\right\rfloor+1}^{n} \sum_{k=0}^{\left\lfloor\frac{j-1}{4 \ell+2}\right\rfloor-1} H_{(4 \ell+2) k+a, j}^{(\ell)} \\
& R_{n, 2}:=\sum_{\ell \geq 1} \sum_{u=0}^{\left\lfloor\frac{n}{4 \ell+2}\right\rfloor} \sum_{\substack{a, b \in[4 \ell+2] \\
a<b}} H_{u(4 \ell+2)+a, u(4 \ell+2)+b}^{(\ell)} \\
& R_{n, 3}:=\sum_{\ell \geq 1} \sum_{v=1}^{\left\lfloor\frac{n}{4 \ell+2}\right\rfloor-1} \sum_{\substack{a, b \in[4 \ell+2] \\
0 \leq a-b \leq(2 \ell+1)-1}}\left(H_{a, v(4 \ell+2)+b}^{(\ell)}+H_{b, v(4 \ell+2)+a}^{(\ell)}\right) \\
& R_{n, 4}=\sum_{\ell \geq 1} \sum_{\substack{a, b \in[4 \ell+2] \\
(2 \ell+1) \leq a-b \leq(4 \ell+2)-1}} \sum_{v=1}^{\left\lfloor\frac{n}{4 \ell+2}\right\rfloor-1}\left(H_{a,(v+1)(4 \ell+2)+b}^{(\ell)}+H_{(4 \ell+2)+b, v(4 \ell+2)+a}^{(\ell)}\right)
\end{aligned}
$$

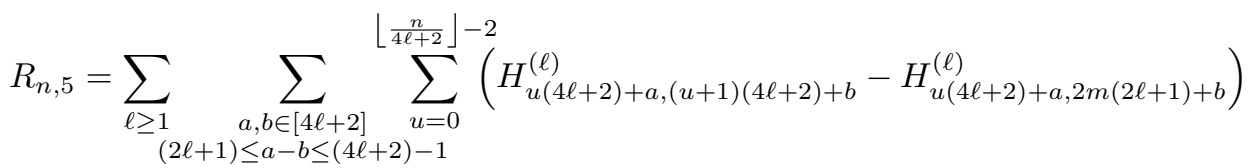

$$
\begin{aligned}
& R_{n, 6}=\sum_{\ell \geq 1} \sum_{\substack{a, b \in[4 \ell+2] \\
(2 \ell+1) \leq a-b \leq(4 \ell+2)-1}} \sum_{v=1}^{\left\lfloor\frac{n}{4 \ell+2}\right\rfloor}\left(H_{b, v(4 \ell+2)+a}^{(\ell)}-H_{v(4 \ell+2)+b, v(4 \ell+2)+a}^{(\ell)}\right),
\end{aligned}
$$

with

$$
\begin{aligned}
H_{i, j}^{(\ell)}:= & h\left(f_{\ell}\left(V_{i, \ell}\right), f_{\ell}\left(V_{j, \ell}\right)\right)-\mathbb{E}\left[h\left(f_{\ell}\left(V_{i, \ell}\right), f_{\ell}\left(V_{j, \ell}\right)\right)\right] \\
& -\left(h\left(f_{\ell-1}\left(V_{i, \ell-1}\right), f_{\ell-1}\left(V_{j, \ell-1}\right)\right)-\mathbb{E}\left[h\left(f_{\ell-1}\left(V_{i, \ell-1}\right), f_{\ell-1}\left(V_{j, \ell-1}\right)\right)\right]\right) .
\end{aligned}
$$


Let us make some comments on the decomposition (1.19). The terms associated to terms of the form $\mathbb{E}\left[h\left(f_{\ell}\left(V_{k, \ell}\right), f_{\ell}\left(V_{0, \ell}^{\prime}\right)\right) \mid V_{k, \ell}\right]$, that is, the first two terms of the right hand side of (1.19), are partial sums of a strictly stationary sequence.

In all our application to limit theorems, the term $R_{n}:=R_{n, 1,1}+R_{n, 1,2}+$ $\sum_{k=2}^{6} R_{n, k}$ will have a negligible contribution: for the law of large numbers, $\left(R_{n} / n^{1+1 / p}\right)_{n \geq 1}$ converges to 0 almost surely, for the law of the iterated logarithms, the $p$-th moment $\sup _{n \geq 1}\left|R_{n}\right| /\left(n^{3 / 2} \mathrm{LL}(n)\right)$ can be bounded in an easier way than for $U$-statistics and for the central limit theorem, $\left(R_{n} / n^{3 / 2}\right)_{n \geq 1}$ converges to 0 in probability.

For the strong law of large numbers, one can show that the term associated to the degenerated $U$-statistics normalized by $n^{-2 / p}$ goes to 0 almost surely, hence its contribution is negligible with respect to the term associated with the stationary sequence. For the law of the iterated logarithms and the central limit theorem, the main contribution is made by the term associated to a stationary sequence.

Let us now explain the origin of the terms involved in (1.19). First of all, we decompose the original centered $U$-statistics as a series (indexed by $\ell$ ) of $U$-statistics. Each term is a $U$-statistics whose data is a function of $2 \ell+1$ independent random variables. Up to some technical steps, these can be decomposed as a sum of $U$-statistics whose data consists of independent identically distributed vectors.

Notice that in Hsing and Wu (2004), the author use a similar approach in the derivation of a central limit theorem, that is, an approximation by $U$-statistics of independent data combined with Hoeffding decomposition. The main difference is that the obtained decomposition in the aforementioned paper gives a $U$-statistic having a more general form than the one in (1.5), since the function $h$ is allowed to vary with the indexes $i$ and $j$. The linear term is not stationary, not even in the case of constant weights. This is not an issue in establishing a central limit theorem, as the involved linear terms are approximated by functions of $\ell$-dependent sequences and one can use a central limit theorem for arrays. However, in the context of the law of large numbers and the bounded law of the iterated logarithms for $U$ statistics with Bernoulli data, one would like to use the results corresponding to $U$-statistics of i.i.d. data, like Proposition 1.2 and Theorem 2.5 in Arcones and Giné (1995). It is likely that some results could be obtained for more general Hoeffding's decompositions, that is, involving $U$-statistics with varying kernel, but it seems to be beyond the scope of this paper.

1.4. Law of large numbers. We first present a result on the Marcinkievicz law of large numbers for $U$-statistics of i.i.d. data. In order to extend it to the context of functional of i.i.d., we need a control on a maximal function.

Proposition 1.2 (Marcinkievicz-Zygmund law of large numbers). Let $\left(\varepsilon_{i}\right)_{i \in \mathbb{Z}}$ be an i.i.d. sequence with values in $\mathbb{R}^{d}$ and let $h: \mathbb{R}^{d} \times \mathbb{R}^{d} \rightarrow \mathbb{R}$ be a measurable function. For $1 \leq p<2$, define

$$
M_{p}:=\sup _{n \geq 1} \frac{1}{n^{2 / p}}\left|U_{n}^{\text {ind }}\left(h,\left(\varepsilon_{i}\right)_{i \in \mathbb{Z}}\right)\right| .
$$

Assume that $\mathbb{E}\left[\left|h\left(\varepsilon_{0}, \varepsilon_{1}\right)\right|^{p}\right]$ is finite and that for all $x \in \mathbb{R}^{d}, \mathbb{E}\left[h\left(\varepsilon_{0}, x\right)\right]=0$. Then the following statements hold:

(1) the sequence $\left(n^{-2 / p}\left|U_{n}^{\text {ind }}\left(h,\left(\varepsilon_{i}\right)_{i \in \mathbb{Z}}\right)\right|\right)_{n \geq 1}$ converges to zero almost surely; 
(2) for any positive $x$,

$$
x^{p} \mathbb{P}\left\{M_{p}>x\right\} \leq \kappa_{p} \mathbb{E}\left[\left|h\left(\varepsilon_{0}, \varepsilon_{1}\right)\right|^{p}\right],
$$

where $\kappa_{p}$ is bigger than 1 and depends only on $p$.

Corollary 1.3. Let $\left(\varepsilon_{i}\right)_{i \in \mathbb{Z}}$ be an i.i.d. sequence with values in $\mathbb{R}^{d}$ and let $h: \mathbb{R}^{d} \times$ $\mathbb{R}^{d} \rightarrow \mathbb{R}$ be a measurable function. For $1 \leq p<2$, define

$$
M_{p}:=\sup _{n \geq 1} \frac{1}{n^{1+1 / p}}\left|U_{n}^{\text {ind }}\left(h,\left(\varepsilon_{i}\right)_{i \in \mathbb{Z}}\right)\right| .
$$

Assume that $\mathbb{E}\left[\left|h\left(\varepsilon_{0}, \varepsilon_{1}\right)\right|^{p}\right]$ is finite and $\mathbb{E}\left[h\left(\varepsilon_{0}, \varepsilon_{1}\right)\right]=0$. Then the following statements hold:

(1) the sequence $\left(n^{-1-1 / p}\left|U_{n}^{\text {ind }}\left(h,\left(\varepsilon_{i}\right)_{i \in \mathbb{Z}}\right)\right|\right)_{n \geq 1}$ converges to zero almost surely;

(2) for any positive $x$,

$$
x^{p} \mathbb{P}\left\{M_{p}>x\right\} \leq \kappa_{p} \mathbb{E}\left[\left|h\left(\varepsilon_{0}, \varepsilon_{1}\right)\right|^{p}\right],
$$

where $\kappa_{p}$ is bigger than 1 and depends only on $p$.

The previous Proposition gives a control in terms of the weak- $\mathbb{L}^{p}$-semi-norm, defined as $\|X\|_{p, w}:=\sup _{x>0}\left(x^{p} \mathbb{P}\{|X|>x\}\right)^{1 / p}, p>1$. However, the triangular inequality may fail, which is not convenient in view of the decomposition obtained in Proposition 1.1. For this reason, we introduce the weak- $\mathbb{L}^{p}$-norm, defined as

$$
\|X\|_{p, \infty}:=\sup _{A: \mathbb{P}(A)>0} \mathbb{P}(A)^{1 / p-1} \mathbb{E}\left[|X| \mathbf{1}_{A}\right] .
$$

Notice that for all fixed $p>1$, there exists constants $c_{p}$ and $C_{p}$ such that for all random variable $X, c_{p}\|X\|_{p, w} \leq\|X\|_{p, \infty} \leq C_{p}\|X\|_{p, w}$. Moreover, the observation that for any sequence of non-negative random variables $\left(Y_{n}\right)_{n \geq 1}$,

$$
\left\|\sup _{n \geq 1} Y_{n}\right\|_{p, w}^{p} \leq \sum_{n \geq 1}\left\|Y_{n}\right\|_{p, w}^{p}
$$

will be useful in the sequel.

We are now in position to present our first result, which gives a control on the maximal function of a $U$-statistic whose data is a functional of an i.i.d. sequence.

Theorem 1.4. Let $\left(\varepsilon_{u}\right)_{u \in \mathbb{Z}}$ be an i.i.d. sequence, $f: \mathbb{R}^{\mathbb{Z}} \rightarrow \mathbb{R}$ and $h: \mathbb{R} \times \mathbb{R} \rightarrow \mathbb{R}$ be measurable functions. Let $p \in[1,2)$. Then

$$
\begin{array}{r}
\left\|\sup _{n \geq 1} \frac{1}{n^{1+1 / p}}\left|U_{n}\left(h, f,\left(\varepsilon_{u}\right)_{u \in \mathbb{Z}}-\mathbb{E}\left[U_{n}\left(h, f,\left(\varepsilon_{u}\right)_{u \in \mathbb{Z}}\right)\right]\right)\right|\right\|_{p, \infty} \\
\leq c_{p}\left(\theta_{0, p}+\sum_{\ell \geq 1} \ell^{1-1 / p} \theta_{\ell, p}\right) .
\end{array}
$$

The second result of this Subsection is a Marcinkievicz law of large numbers.

Theorem 1.5 (Marcinkievicz law of large numbers). Let $\left(\varepsilon_{u}\right)_{u \in \mathbb{Z}}$ be an i.i.d. sequence, $f: \mathbb{R}^{\mathbb{Z}} \rightarrow \mathbb{R}$ and $h: \mathbb{R} \times \mathbb{R} \rightarrow \mathbb{R}$ be measurable functions. Let $p \in[1,2)$. Suppose that

$$
\sum_{\ell \geq 0} \ell^{1-1 / p} \theta_{\ell, p}<+\infty
$$


Then the following almost sure convergence holds:

$$
\frac{1}{n^{1+1 / p}}\left|U_{n}\left(h, f,\left(\varepsilon_{u}\right)_{u \in \mathbb{Z}}-\mathbb{E}\left[U_{n}\left(h, f,\left(\varepsilon_{u}\right)_{u \in \mathbb{Z}}\right)\right]\right)\right| \rightarrow 0 .
$$

A law of large numbers has been obtained in Borovkova et al. (2001), Theorem 6 . They considered the classical law of large numbers $(p=1)$ but for functions of a $\beta$-mixing process. When applied to a Lipschitz-continuous kernel and a function of an i.i.d. process, their condition requires

$$
\sum_{\ell \geq 1}\left\|f\left(\left(\varepsilon_{-u}\right)_{u \in \mathbb{Z}}\right)-\mathbb{E}\left[f\left(\left(\varepsilon_{-u}\right)_{u \in \mathbb{Z}}\right) \mid \varepsilon_{-\ell}, \ldots, \varepsilon_{\ell}\right]\right\|_{1}<+\infty .
$$

When $f\left(\left(\varepsilon_{-u}\right)_{u \in \mathbb{Z}}\right)$ is a linear process with Bernoulli innovations, that is, $f\left(\left(\varepsilon_{-u}\right)_{u \in \mathbb{Z}}\right)=\sum_{i \in \mathbb{Z}} a_{i} \varepsilon_{-i}$ and $\mathbb{P}\left\{\varepsilon_{0}=1\right\}=\mathbb{P}\left\{\varepsilon_{0}=-1\right\}=1 / 2$, this is equivalent, by Marcinkievicz-Zygmund inequality, to

$$
\sum_{\ell \geq 1}\left(\sum_{i \in \mathbb{Z},|i| \geq \ell} a_{i}^{2}\right)^{1 / 2}<+\infty,
$$

while our condition read $\sum_{i \in \mathbb{Z}}\left|a_{i}\right|<+\infty$ and is less restrictive. However, in Borovkova et al. (2001), the case of functional of $\beta$-mixing process and of a not necessarily continuous kernel is treated there and not in the present paper.

1.5. Bounded law of the iterated logarithms. Let us present our next result concerning the bounded law of the iterated logarithms. Like in the independent case, one can control the moments of order $p \in(1,2)$ of the maximal function of $U$-statistic with the normalisation $n^{3 / 2}$ LL $(n)$.

Theorem 1.6. Let $\left(\varepsilon_{u}\right)_{u \in \mathbb{Z}}$ be an i.i.d. sequence, $f: \mathbb{R}^{\mathbb{Z}} \rightarrow \mathbb{R}$ and $h: \mathbb{R} \times \mathbb{R} \rightarrow \mathbb{R}$ be measurable functions. Let $p \in[1,2)$. For all $1 \leq p<2$, the following inequality holds:

$$
\begin{array}{r}
\left\|\sup _{n \geq 1} \frac{1}{n^{3 / 2} \sqrt{\mathrm{LL}(n)}}\left|U_{n}\left(h, f,\left(\varepsilon_{u}\right)_{u \in \mathbb{Z}}\right)-\mathbb{E}\left[U_{n}\left(h, f,\left(\varepsilon_{u}\right)_{u \in \mathbb{Z}}\right)\right]\right|\right\|_{p} \\
\leq c_{p}\left(\theta_{0,2}+\sum_{\ell \geq 1} \ell^{1 / 2} \theta_{\ell, 2}\right),
\end{array}
$$

where $c_{p}$ depends only on $p$.

1.6. Central limit theorem. Let us present our result concerning the central limit theorem. We will make essentially three assumptions on the dependence of our $U$ statistic. The first and second one involve the coefficients $\delta_{., 2}$ and $\theta_{\ell, 1}$ respectively and the third condition is imposed is summability of the family of covariances of a strictly stationary sequence which comes from the generalized Hoeffding decomposition of Proposition 1.1.

Theorem 1.7. Let $h: \mathbb{R}^{2} \rightarrow \mathbb{R}$ be a measurable function, $f: \mathbb{R}^{\mathbb{Z}} \rightarrow \mathbb{R}$ be a measurable function and $\left(\varepsilon_{u}\right)_{u \in \mathbb{Z}}$ be an i.i.d. sequence. Let

$$
X_{j}:=f\left(\left(\varepsilon_{j-k}\right)_{k \in \mathbb{Z}}\right), \quad U_{n}:=\sum_{1 \leq i<j \leq n} h\left(X_{i}, X_{j}\right) .
$$


Suppose that

$$
\sum_{\ell \geq 0} \ell^{1 / 2} \theta_{\ell, 2}<+\infty ; \quad \sum_{\ell \geq 0} \ell^{2} \theta_{\ell, 1}<+\infty
$$

and that

$$
\sum_{k \in \mathbb{Z}}\left|\operatorname{Cov}\left(Y_{0}, Y_{k}\right)\right|<+\infty,
$$

where the random variable $Y_{k}$ is defined by the following $\mathbb{L}^{2}$-convergence

$$
Y_{k}=\lim _{\ell \rightarrow+\infty} \mathbb{E}\left[h\left(f_{\ell}\left(V_{k, \ell}\right), f_{\ell}\left(V_{0, \ell}^{\prime}\right)\right) \mid V_{k, \ell}\right]-\mathbb{E}\left[h\left(f_{\ell}\left(V_{k, \ell}\right), f_{\ell}\left(V_{0, \ell}^{\prime}\right)\right)\right]
$$

and $V_{k, \ell}=\left(\varepsilon_{u}\right)_{u=k-\ell}^{k+\ell}, V_{k, \ell}^{\prime}=\left(\varepsilon_{u}^{\prime}\right)_{u=k-\ell}^{k+\ell}$, where $\left(\varepsilon_{u}^{\prime}\right)_{u \in \mathbb{Z}}$ is an independent copy of $\left(\varepsilon_{u}\right)_{u \in \mathbb{Z}} \cdot$

Then the following convergence in distribution holds:

$$
\frac{1}{n^{3 / 2}}\left(U_{n}-\mathbb{E}\left[U_{n}\right]\right) \rightarrow N\left(0, \sigma^{2}\right)
$$

where

$$
\sigma^{2}:=\sum_{k \in \mathbb{Z}} \operatorname{Cov}\left(Y_{0}, Y_{k}\right) .
$$

Let us give some comments about this results and how it relates with existing results.

Remark 1.8. It is not clear to us whether the condition (1.33) guarantees (1.34). Nevertheless, conditions of the type

$$
\sum_{k \in \mathbb{Z}}\left\|\mathbb{E}\left[Y_{k} \mid \sigma\left(\varepsilon_{u}, u \leq 0\right)\right]-\mathbb{E}\left[Y_{k} \mid \sigma\left(\varepsilon_{u}, u \leq-1\right)\right]\right\|_{2}<\infty
$$

guarantee (1.34) and are not too hard to check for the choices of kernel involved in Subsection 1.7. Note also that the convergence of $\sum_{\ell \geq 1} \theta_{\ell, 2}$ guarantees that $Y_{k}$ admits the simpler form

$$
\begin{aligned}
Y_{k}=\mathbb{E}\left[h \left(f\left(\left(\varepsilon_{k-u}\right)_{u \in \mathbb{Z}}\right), f(\right.\right. & \left.\left.\left.\left(\varepsilon_{-u}^{\prime}\right)_{u \in \mathbb{Z}}\right)\right) \mid \sigma\left(\varepsilon_{u}, u \in \mathbb{Z}\right)\right] \\
& -\mathbb{E}\left[h\left(f\left(\left(\varepsilon_{k-u}\right)_{u \in \mathbb{Z}}\right), f\left(\left(\varepsilon_{-u}^{\prime}\right)_{u \in \mathbb{Z}}\right)\right)\right] .
\end{aligned}
$$

Condition (1.38) can be checked under the assumption that

$$
\sum_{k \in \mathbb{Z}}\left\|Y_{k}-Y_{k}^{*}\right\|_{2}<\infty
$$

where $Y_{k}^{*}$ is a random variable defined as $Y_{k}$, but the occurence of $\varepsilon_{0}$ is replaced by $\varepsilon_{0}^{\prime \prime}$, where $\varepsilon_{0}^{\prime \prime}$ has the same law as $\varepsilon_{0}$ and is independent of the $\sigma$-algebra generated by the sequences $\left(\varepsilon_{u}\right)_{u \in \mathbb{Z}}$ and $\left(\varepsilon_{u}^{\prime}\right)_{u \in \mathbb{Z}}$.

Remark 1.9. In the context of Theorem 1.7, it is possible that the variance $\sigma^{2}$ equals 0 . In this case, the normalization should change and a deeper analysis is required in order to identify the limiting process.

Remark 1.10. It does not seem to be an easy task to compare the condition of Theorem 1.7 with those in Hsing and Wu (2004) in full generality. One way to 
measure the dependence in the latter paper is given (in the case where the weights are all equal to one) by the coefficients

$$
\delta_{\ell}:=\sup _{j \geq 0}\left\|h\left(\left(\varepsilon_{-u}\right)_{u \in \mathbb{Z}},\left(\varepsilon_{j-u}\right)_{u \in \mathbb{Z}}\right)-h\left(f_{\ell}\left(V_{0, \ell}\right), f_{\ell}\left(V_{j, \ell}\right)\right)\right\|_{2} .
$$

A sufficient condition (which follows from a combination of Theorem 3 and Proposition 4) for the central limit theorem is

$$
\sum_{i \geq 1} \sup _{\ell \geq i} \delta_{\ell}<+\infty
$$

In a particular case, this condition can be compared with ours. Let $h:(x, y) \mapsto x+y$ and $\left(X_{j}\right)_{j \geq 1}$ be a causal linear process:

$$
X_{j}:=\sum_{i \geq 0} a_{i} \varepsilon_{j-i}
$$

where $\left(\varepsilon_{u}\right)_{u \in \mathbb{Z}}$ is i.i.d. centered and square integrable and $\left(a_{i}\right)_{i \in \mathbb{Z}}$ is square summable, conditon (1.42) reads

$$
\sum_{i \geq 1} \sqrt{\sum_{\ell \geq i} a_{\ell}^{2}}<+\infty
$$

(see Theorem 9). With the same kernel and definition of $\left(X_{j}\right)_{j \geq 1}$, our condition is more restrictive, since the convergence of $\sum_{\ell \geq 0} \ell^{2} \theta_{\ell, 1}$ implies that of $\sum_{\ell \geq 0} \ell^{2} a_{\ell}$.

Nevertheless, our result deals with not necessarily causal process.

Remark 1.11. A central limit theorem has been obtained in Borovkova et al. (2001), Theorem 7, for functions of a $\beta$-mixing process. When applied to a Lipschitzcontinuous kernel and a function of an i.i.d. process, their condition requires

$$
\sum_{\ell \geq 1} \ell^{2}\left\|f\left(\left(\varepsilon_{-u}\right)_{u \in \mathbb{Z}}\right)-\mathbb{E}\left[f\left(\left(\varepsilon_{-u}\right)_{u \in \mathbb{Z}}\right) \mid \varepsilon_{-\ell}, \ldots, \varepsilon_{\ell}\right]\right\|_{1}<+\infty .
$$

When $f\left(\left(\varepsilon_{-u}\right)_{u \in \mathbb{Z}}\right)$ is a linear process with Bernoulli innovations, that is, $f\left(\left(\varepsilon_{-u}\right)_{u \in \mathbb{Z}}\right)=\sum_{i \in \mathbb{Z}} a_{i} \varepsilon_{-i}$, this is equivalent to

$$
\sum_{\ell \geq 1} \ell^{2}\left(\sum_{i \in \mathbb{Z},|i| \geq \ell} a_{i}^{2}\right)^{1 / 2}<+\infty,
$$

while our condition reads $\sum_{\ell \geq 1} \ell^{2}\left(\left|a_{\ell}\right|+\left|a_{-\ell}\right|\right)<+\infty$ and is less restrictive. However, in Borovkova et al. (2001), the case of functional of $\beta$-mixing process and of a not necessarily continuous kernel is treated there and not in the present paper.

Remark 1.12. In Beutner and Zähle (2012), an approach based on empirical processes has been done in order to derive a central limit theorem for $U$-statistics of dependent data. As mentioned in Example 3.8, the case of function of i.i.d. processes can be treated, since the convergence of empirical processes of functions of an i.i.d. sequence has been treated in Wu (2008). However, in the later paper, the condition on the weak dependence relies on the physical dependence measure of $\mathbf{1}\left\{f\left(\left(\varepsilon_{i-u}\right)_{u \in \mathbb{Z}}\right) \leq t\right\}$ and this does not seem to be comparable with our assumptions.

1.7. Applications. In this Subsection, we give examples of kernels $h$ for which the measure of dependence defined by (1.8) can be estimated. 
1.7.1. Uniformly continuous kernel. Let $h: \mathbb{R}^{2} \rightarrow \mathbb{R}$ be a measurable function. We assume that there exists a non-negative function $\omega: \mathbb{R}_{+} \rightarrow \mathbb{R}_{+}$which is increasing, satisfies $\omega(0)=0$ and for each $x, x^{\prime}, y, y^{\prime} \in \mathbb{R}$,

$$
\left|h(x, y)-h\left(x^{\prime}, y^{\prime}\right)\right| \leq \omega\left(\left|x-x^{\prime}\right|\right)+\omega\left(\left|y-y^{\prime}\right|\right) .
$$

Then the following inequality holds:

$$
\begin{aligned}
& \left\|h\left(f_{\ell}\left(V_{0, \ell}\right), f_{\ell}\left(V_{j, \ell}\right)\right)-h\left(f_{\ell-1}\left(V_{0, \ell-1}\right), f_{\ell-1}\left(V_{j, \ell-1}\right)\right)\right\|_{p} \\
& \quad \leq\left\|\omega\left(\left|f_{\ell}\left(V_{0, \ell}\right)-f_{\ell-1}\left(V_{0, \ell-1}\right)\right|\right)\right\|_{p}+\left\|\omega\left(\left|f_{\ell}\left(V_{j, \ell}\right)-f_{\ell-1}\left(V_{j, \ell-1}\right)\right|\right)\right\|_{p} .
\end{aligned}
$$

Since $\left(V_{j, \ell}, V_{j, \ell-1}\right)$ has the same distribution as $\left(V_{0, \ell}, V_{0, \ell-1}\right)$, the two terms of the right hand side of (1.48) are equal hence

$$
\theta_{\ell, p} \leq 2\left\|\omega\left(\left|f_{\ell}\left(V_{0, \ell}\right)-f_{\ell-1}\left(V_{0, \ell-1}\right)\right|\right)\right\|_{p} .
$$

In particular, if $h$ is $\alpha$-Hölder continuous for some $\alpha \in(0,1)$, we can choose $\omega: t \mapsto c t^{\alpha}$ for some constant $c$ and in this case, the estimate (1.49) becomes

$$
\theta_{\ell, p} \leq 2 c\left\|f_{\ell}\left(V_{0, \ell}\right)-f_{\ell-1}\left(V_{0, \ell-1}\right)\right\|_{p \alpha}^{\alpha},
$$

which can be rewritten as

$$
\theta_{\ell, p} \leq 2 c\left\|\mathbb{E}\left[X_{0} \mid V_{0, \ell}\right]-\mathbb{E}\left[X_{0} \mid V_{0, \ell-1}\right]\right\|_{p \alpha}^{\alpha} .
$$

1.7.2. Variance estimation. Consider the kernel $h: \mathbb{R}^{2} \rightarrow \mathbb{R}$ defined by $h(x, y):=$ $(x-y)^{2} / 2$. The associated $U$-statistic is (after normalization) the classical variance estimator. For this choice of kernel, one can estimate the measure of dependence defined by (1.8). One needs to control the $\mathbb{L}^{p}$-norm of the difference of the square of two random variables $Y$ and $Z$. Since

$$
\left\|Y^{2}-Z^{2}\right\|_{p}^{p}=\mathbb{E}\left[|Y-Z|^{p}|Y+Z|^{p}\right] \leq \mathbb{E}\left[|Y-Z|^{2 p}\right]^{1 / 2} \mathbb{E}\left[|Y+Z|^{2 p}\right]^{1 / 2}
$$

we derive that

$$
\left\|Y^{2}-Z^{2}\right\|_{p} \leq\|Y-Z\|_{2 p}\left(\|Y\|_{2 p}+\|Z\|_{2 p}\right) .
$$

We use this for a fixed $\ell \geq 1$ to $Y=f_{\ell}\left(V_{0, \ell}\right)-f_{\ell}\left(V_{j, \ell}\right)$ and $Z=f_{\ell-1}\left(V_{0, \ell-1}\right)-$ $f_{\ell-1}\left(V_{j, \ell-1}\right)$. Accounting the following bounds (which are a consequence of stationarity):

$$
\|Y\|_{2 p}+\|Z\|_{2 p} \leq 2\left\|f_{\ell}\left(V_{0, \ell}\right)\right\|_{2 p}+2\left\|f_{\ell}\left(V_{0, \ell-1}\right)\right\|_{2 p} \leq 4\left\|X_{0}\right\|_{2 p},
$$

we get that

$$
\theta_{\ell, p} \leq 2\left\|X_{0}\right\|_{2 p}\left\|\mathbb{E}\left[X_{0} \mid V_{0, \ell}\right]-\mathbb{E}\left[X_{0} \mid V_{0, \ell-1}\right]\right\|_{p} .
$$

1.7.3. Correlation integral. Correlation integral is defined for a sequence of random variables $\left(X_{i}\right)_{i \geq 1}$ and $t>0$ as $N_{n, t}:=\sum_{1 \leq i \neq j \leq n} 1\left\{\left|X_{i}-X_{j}\right| \leq t\right\}$. It plays an important role in the study of the dynamical systems, see for instance Denker and Keller (1986); Serinko (1994); Wolff (1990). Since the summand is symmetric in $i$ and $j$ and vanishes for $i=j$, the correlation integral $N_{n, t}$ may be expressed as a $U$-statistic with kernel $h:(x, y) \in \mathbb{R}^{2} \mapsto \mathbf{1}\{|x-y| \leq t\}$. Under some conditions on the density of $f_{\ell}\left(V_{0, \ell}\right)$ and the dependence of the sequence $\left(X_{i}\right)_{i \geq 1}$, one can derive bounds for $\theta_{\ell, p}$ and the obtained limit theorems translate in the following way. 
Theorem 1.13. Let $\left(X_{i}\right)_{i \geq 0}$ be a sequence such that $X_{i}=f\left(\left(\varepsilon_{i-u}\right)_{u \in \mathbb{Z}}\right)$ for some measurable function $f: \mathbb{R}^{\mathbb{Z}} \rightarrow \mathbb{R}$. Let $t>0$ and $N_{n, t}=$ $\sum_{1 \leq i \neq j \leq n} 1\left\{\left|X_{i}-X_{j}\right| \leq t\right\}$. Suppose that for each $\ell$ and $j \geq 1$, the random variable $\mathbb{E}\left[X_{0} \mid \sigma\left(\varepsilon_{u},-\ell \leq u \leq \ell\right)\right]-\mathbb{E}\left[X_{j} \mid \sigma\left(\varepsilon_{u}, j-\ell \leq u \leq j+\ell\right)\right]$ has a density $g_{\ell, j}$ and that for some $r>1$, $\sup _{\ell, j \geq 1}\left\|g_{\ell, j}\right\|_{\mathbb{L}^{r}(\mathbb{R})}$ is finite. Let $\pi_{\ell}:=\mathbb{E}\left[X_{0} \mid V_{0, \ell}\right]-$ $\mathbb{E}\left[X_{0} \mid V_{0, \ell-1}\right]$, where $V_{0, k}=\left(\varepsilon_{u}\right)_{u=-k}^{k}$.

(1) Strong law of large numbers. If for some $p \in[1,2)$ and $q \geq 1$, the series $\sum_{\ell \geq 1} \ell^{1-1 / p}\left\|\pi_{\ell}\right\|_{q}^{\frac{q}{p} \frac{r}{q(r-1)+r}}$ converges, then

$$
\left\|\sup _{n \geq 1} \frac{1}{n^{1+1 / p}}\left|N_{n, t}-\mathbb{E}\left[N_{n, t}\right]\right|\right\|_{p} \leq C_{p}
$$

and $\frac{1}{n^{1+1 / p}}\left|N_{n, t}-\mathbb{E}\left[N_{n, t}\right]\right| \rightarrow 0$ almost surely.

(2) Bounded law of the iterated logarithms. Suppose that $\sum_{\ell \geq 1} \ell^{1 / 2}\left\|\pi_{\ell}\right\|_{q}^{\frac{q}{2} \frac{r}{q(r-1)+r}}$ is finite for some $q \geq 1$. Then for each $p \in[1,2)$,

$$
\left\|\sup _{n \geq 1} \frac{1}{n^{3 / 2} \sqrt{\mathrm{LL}(n)}}\left|N_{n, t}-\mathbb{E}\left[N_{n, t}\right]\right|\right\|_{p}
$$

is finite.

(3) Central limit theorem. Let $q \geq 1$. Suppose that the series $\sum_{\ell \geq 1} \ell^{1 / 2}\left\|\pi_{\ell}\right\|_{q}^{\frac{q}{2} \frac{r}{q(r-1)+r}}$ and $\sum_{\ell \geq 1} \ell^{2}\left\|\pi_{\ell}\right\|_{q}^{q \frac{r}{q(r-1)+r}}$ are convergent. Assume furthermore that for each $k, Z_{k}-Z_{k}^{\prime}$ has a density $g_{k}$ such that $\sup _{k \geq 1}\left\|g_{k}\right\|_{\mathbb{L}^{r}(\mathbb{R})}$ is finite, where

$$
Z_{k}=\mathbb{E}\left[\mathbf{1}\left\{\left|f\left(\left(\varepsilon_{k-u}\right)_{u \in \mathbb{Z}}\right)-f\left(\left(\varepsilon_{-u}^{\prime}\right)_{u \in \mathbb{Z}}\right)\right| \leq t\right\}\right]
$$

and $Z_{k}^{\prime}=\mathbb{E}\left[\mathbf{1}\left\{\left|f\left(\left(\varepsilon_{k-u}^{*}\right)_{u \in \mathbb{Z}}\right)-f\left(\left(\varepsilon_{-u}^{\prime}\right)_{u \in \mathbb{Z}}\right)\right| \leq t\right\}\right]$, where $\varepsilon_{i}^{*}=\varepsilon_{i}$ if $i \neq 0, \varepsilon_{0}^{*}=\varepsilon_{0}^{\prime \prime}$ and $\left(\varepsilon_{u}\right)_{u \in \mathbb{Z}},\left(\varepsilon_{u}^{\prime}\right)_{u \in \mathbb{Z}}$ and $\varepsilon_{0}^{\prime \prime}$ are independent and $\varepsilon_{u}, \varepsilon_{u}^{\prime}$ and $\varepsilon_{u}^{\prime \prime}$ have the same distribution. We finally assume that $\sum_{k \in \mathbb{Z}}\left\|Z_{k}-Z_{k}^{\prime}\right\|_{q}^{\frac{r q}{2(q r+r-1)}}$ is finite.

Then there exists $\sigma$ such that the convergence in distribution

$$
\frac{N_{n, t}-\mathbb{E}\left[N_{n, t}\right]}{n^{3 / 2}} \rightarrow \sigma N
$$

takes places, where $N$ is a standard normal random variable.

\section{Proofs}

2.1. Proof of the generalised Hoeffding's decomposition. Let us explain the idea of proof of Proposition 1.1. First, we write $U_{n}\left(h, f,\left(\varepsilon_{i}\right)_{i \in \mathbb{Z}}\right)$ as a series (index by $\ell$ ) of $U$-statistics. For the term of index $\ell$, the data of the corresponding $U$-statistic is a function of $2 \ell+1$ i.i.d. random variables. We then decompose this a sum of $4(2 \ell+1) U$-statistics of independent data.

We divide the proof in two steps:

(1) First we treat the case where $X_{j}=f\left(\left(\varepsilon_{u}\right)_{u=j-\ell}^{j+\ell}\right)$ and $h: \mathbb{R} \times \mathbb{R} \rightarrow \mathbb{R}$ a symmetric function. 
(2) Then we write the mentioned $U$-statistic a sum of $U$-statistics tractable with the work of step 1 , plus a remainder term.

Step 1: we decompose a $U$-statistic whose data is a function of $2 \ell+1$ i.i.d. random variables as a sum of $4 \ell+2 U$-statistics of i.i.d. data plus remainder terms.

Lemma 2.1. Let $\ell \geq 1$ be an integer, $h: \mathbb{R}^{2 \ell+1} \times \mathbb{R}^{2 \ell+1} \rightarrow \mathbb{R}$ be a measurable function, let $\left(\varepsilon_{u}\right)_{u \in \mathbb{Z}}$ be an i.i.d. real-valued sequence and $\left(\varepsilon_{u}^{\prime}\right)_{u \in \mathbb{Z}}$ an independent copy of $\left(\varepsilon_{u}\right)_{u \in \mathbb{Z}}$. Define $U_{n}\left(h,\left(\varepsilon_{u}\right)_{u \in \mathbb{Z}}\right):=\sum_{1 \leq i<j \leq n} h\left(V_{i}, V_{j}\right)=\sum_{1 \leq i<j \leq n} H_{i, j}$, where $V_{j}=\left(\varepsilon_{u}\right)_{u=j-\ell}^{j+\ell}$ and $H_{i, j}:=h\left(V_{i}, V_{j}\right)$.

Then

$$
U_{n}\left(h,\left(\varepsilon_{u}\right)_{u \in \mathbb{Z}}\right)=\sum_{a, b \in[4 \ell+2]} U_{\left\lfloor\frac{n}{4 \ell+2}\right\rfloor}^{\text {ind }}\left(h_{a, b},\left(\varepsilon_{i}^{a, b}\right)\right)+\sum_{k=1}^{6} R_{n, k},
$$

where

- the function $h_{a, b}: \mathbb{R}^{4 \ell+2} \times \mathbb{R}^{4 \ell+2} \rightarrow \mathbb{R}$ is defined for $0 \leq a-b \leq 2 \ell$ by

$$
\begin{aligned}
& h_{a, b}\left(\left(x_{i}\right)_{i=1}^{4 \ell+2},\left(y_{j}\right)_{j=1}^{4 \ell+2}\right) \\
& \quad:=h\left(\left(x_{i+a-b}\right)_{i=1}^{2 \ell+1},\left(y_{j}\right)_{j=1}^{2 \ell+1}\right)+h\left(\left(y_{j+a-b}\right)_{j=1}^{2 \ell+1},\left(x_{i}\right)_{i=1}^{2 \ell+1}\right) \\
& \quad \text { and for }(2 \ell+1) \leq a-b \leq(4 \ell+2)-1 \text { by } \\
& h_{a, b}\left(\left(x_{i}\right)_{i=1}^{4 \ell+2},\left(y_{j}\right)_{j=1}^{4 \ell+2}\right) \\
& :=h\left(\left(x_{i+a-b-(2 \ell+1)}\right)_{i=1}^{2 \ell+1},\left(y_{j}\right)_{j=1}^{2 \ell+1}\right)+h\left(\left(y_{j+a-b-(2 \ell+1)}\right)_{j=1}^{2 \ell+1},\left(x_{i}\right)_{i=1}^{2 \ell+1}\right),
\end{aligned}
$$

- the random vectors $\varepsilon_{u}^{a, b}$ are defined by

$$
\begin{gathered}
\varepsilon_{u}^{a, b}:=\left(\varepsilon_{j}\right)_{j=u(4 \ell+2)+b-\ell}^{(u+1)(4 \ell+2)+b-\ell-1}, 0 \leq a-b \leq 2 \ell ; \\
\varepsilon_{u}^{a, b}:=\left(\varepsilon_{j}\right)_{j=u(4 \ell+2)+b+\ell+1}^{(u+1)(4 \ell+2)+b+\ell},(2 \ell+1) \leq a-b \leq(4 \ell+2)-1 ;
\end{gathered}
$$

- the remainder terms are defined (with the convention that $\sum_{u=1}^{-k}=0, k \leq 0$ ) by

$$
\begin{aligned}
& R_{n, 1}=\sum_{1 \leq i<j \leq n} H_{i, j}-\sum_{1 \leq i<j \leq(4 \ell+2)\left\lfloor\frac{n}{4 \ell+2}\right\rfloor} H_{i, j} ; \\
& R_{n, 2}:=\sum_{u=0}^{\left\lfloor\frac{n}{4 \ell+2}\right\rfloor} \sum_{\substack{a, b \in[4 \ell+2] \\
a<b}} H_{u(4 \ell+2)+a, u(4 \ell+2)+b} \\
& R_{n, 3}=\sum_{v=1}^{\left\lfloor\frac{n}{4 \ell+2}\right\rfloor} \sum_{\substack{a, b \in[4 \ell+2] \\
0 \leq a-b \leq(2 \ell+1)-1}}\left(H_{a, v(4 \ell+2)+b}+H_{b, v(4 \ell+2)+a}\right) \\
& R_{n, 4}=\sum_{\substack{a, b \in[4 \ell+2] \\
(2 \ell+1) \leq a-b \leq(4 \ell+2)-1}} \sum_{v=1}^{\left\lfloor\frac{n}{4 \ell+2}\right\rfloor}\left(H_{a,(v+1)(4 \ell+2)+b}+H_{(4 \ell+2)+b, v(4 \ell+2)+a}\right) \text {; }
\end{aligned}
$$




$$
\begin{aligned}
R_{n, 5} & =\sum_{\substack{a, b \in[4 \ell+2] \\
(2 \ell+1) \leq a-b \leq(4 \ell+2)-1}} \sum_{u=0}^{\left\lfloor\frac{n}{4 \ell+2}\right\rfloor-2}\left(H_{u(4 \ell+2)+a,(u+1)(4 \ell+2)+b}-H_{u(4 \ell+2)+a, 2 m(2 \ell+1)+b}\right) ; \\
R_{n, 6} & =\sum_{\substack{a, b \in[4 \ell+2] \\
(2 \ell+1) \leq a-b \leq(4 \ell+2)-1}} \sum_{v=1}^{\left\lfloor\frac{n}{4 \ell+2}\right\rfloor}\left(H_{b, v(4 \ell+2)+a}-H_{v(4 \ell+2)+b, v(4 \ell+2)+a}\right) \cdot(2.11)
\end{aligned}
$$

Proof: Before going into the details of the proof, let us explain the general idea, which will also explain the origin of the remainder terms. First, it turns out that it would be more convenient that $n$ is a multiple of $4 \ell+2$. However, it has no reason to be the case and we should also take into account the difference between $U_{n}$ and $U_{n^{\prime}}$ where $n^{\prime}$ is a multiple of $4 \ell+2$ close to $n$. The control of the difference of these two terms is precisely $R_{n, 1}$. Now that we are reduced to the case where $n$ is a multiple of $4 \ell+2$, we look that the terms $H_{i, j}$ and the remainder of $i$ and $j$ by the Euclidian division by $4 \ell+2$. If these remainder are closed enough, then we can write the corresponding sum as a $U$-statistic whose data are independent vectors of length $4 \ell+2$. If the remainders are too far way, we have to add and substract a term to be reduced to the previous case, and this leads to the definition of $R_{n, k}$, $3 \leq k \leq 6$. If the quotient in the Euclidean division by $4 \ell+1$ of $i$ and $j$ are the same, then the corresponding sum is $R_{n, 2}$.

From the equality $U_{n}\left(h_{2}, f,\left(\varepsilon_{i}\right)_{i \in \mathbb{Z}}\right)=\sum_{1 \leq i<j \leq n} H_{i, j}$ we get by (2.6) that

$$
U_{n}\left(h_{2},\left(\varepsilon_{i}\right)_{i \in \mathbb{Z}}\right)=\sum_{1 \leq i<j \leq(4 \ell+2)\left\lfloor\frac{n}{4 \ell+2}\right\rfloor} H_{i, j}+R_{n, 1} .
$$

For simplicity, let us denote by $I$ the set $[4 \ell+2]$ and $m:=\left\lfloor\frac{n}{4 \ell+2}\right\rfloor$. With these notations and in view of (2.7), the following equality takes place

$$
\begin{aligned}
& \sum_{1 \leq i<j \leq(4 \ell+2)\left\lfloor\frac{n}{4 \ell+2}\right\rfloor} H_{i, j} \\
= & \sum_{0 \leq u<v \leq m-1} \sum_{\substack{a, b \in I \\
0 \leq a-b \leq \leq(2 \ell+1)-1}}\left(H_{u(4 \ell+2)+a, v(4 \ell+2)+b}+H_{u(4 \ell+2)+b, v(4 \ell+2)+a}\right) \\
+ & \sum_{\substack{0 \leq u<v \leq m-1 \\
(2 \ell+1) \leq a-b \leq(4 \ell+2)-1}}\left(H_{u(4 \ell+2)+a, v(4 \ell+2)+b}+H_{u(4 \ell+2)+b, v(4 \ell+2)+a}\right)+R_{n, 2} .
\end{aligned}
$$

Let us treat the first term. For $a, b \in I$ such that $0 \leq a-b \leq 2 \ell$, in view of the definitions (2.2) and (2.4) and the symmetry of $h_{2}$, the following equaliy holds

$$
h_{a, b}\left(\varepsilon_{u}^{a, b}, \varepsilon_{v}^{a, b}\right)=H_{u(4 \ell+2)+a, v(4 \ell+2)+b}+H_{u(4 \ell+2)+b, 2 v(4 \ell+2)+a}
$$


hence

$$
\begin{array}{r}
\sum_{0 \leq u<v \leq m-1} \sum_{\substack{a, b \in I \\
0 \leq a-b \leq(2 \ell+1)-1}}\left(H_{u(4 \ell+2)+a, v(4 \ell+2)+b}+H_{u(4 \ell+2)+b, v(4 \ell+2)+a}\right) \\
=U_{m}^{\text {ind }}\left(h_{a, b},\left(\varepsilon_{u}^{a, b}\right)_{u \in \mathbb{Z}}\right)+R_{n, 3} .
\end{array}
$$

Let us treat the second term in the right hand side of (2.13). Adding and subtracting the terms $H_{u(4 \ell+2)+a,(v+1)(4 \ell+2)+b}$ and $H_{(u+1)(4 \ell+2)+b, v(4 \ell+2)+a}$ gives, after having rewriten the corresponding sums as double sums and exploited a telescoping of the inside sum,

$$
\sum_{0 \leq u<v \leq m-1}\left(H_{u(4 \ell+2)+a, v(4 \ell+2)+b}+H_{u(4 \ell+2)+b, v(4 \ell+2)+a}\right)=S+R_{n, 5}+R_{n, 6},
$$

where

$$
S:=\sum_{0 \leq u<v \leq m-1}\left(H_{u(4 \ell+2)+a,(v+1)(4 \ell+2)+b}+H_{2(u+1)(2 \ell+1)+b, v(4 \ell+2)+a}\right)
$$

We express $S$ as a $U$-statistic of independent data. Noticing that $0 \leq a-(b+(2 \ell+1)) \leq(2 \ell+1)-1$, we are in a similar situation as in the case $0 \leq a-b \leq(2 \ell+1)-1$, with $b$ replaced by $b+(2 \ell+1)$. Therefore, in view of $(2.5),(2.3)$ and $(2.9)$, we obtain that

$$
S=U_{m-1}^{\mathrm{ind}}\left(h_{a, b},\left(\varepsilon_{u}^{a, b}\right)_{u \in \mathbb{Z}}\right)+R_{n, 4} .
$$

Collecting these terms gives

$$
\begin{gathered}
\quad \sum_{\substack{a, b \in I \\
(2 \ell+1) \leq a-b \leq(4 \ell+2)-1}} \sum_{0 \leq u<v \leq m-1}\left(H_{u(4 \ell+2)+a, v(4 \ell+2)+b}+H_{u(4 \ell+2)+b, v(4 \ell+2)+a}\right) \\
=\sum_{\substack{a, b \in I \\
(2 \ell+1) \leq a-b \leq(4 \ell+2)-1}} U_{m-1}^{\mathrm{ind}}\left(h_{a, b},\left(\varepsilon_{u}^{a, b}\right)_{u \in \mathbb{Z}}\right)+R_{n, 4}+R_{n, 5}+R_{n, 6} .
\end{gathered}
$$

We end the proof of Lemma 2.1 by combining the equalities (2.13), (2.15) and (2.18).

Now, we can apply the Hoeffding decomposition to each $U$-statistic of i.i.d. data and rewrite the remainder term in a more tractable form.

Lemma 2.2. Let $\ell \geq 1$ be an integer, $h: \mathbb{R}^{2 \ell+1} \times \mathbb{R}^{2 \ell+1} \rightarrow \mathbb{R}$ be a measurable function, let $\left(\varepsilon_{u}\right)_{u \in \mathbb{Z}}$ be an i.i.d. real-valued sequence and $\left(\varepsilon_{u}^{\prime}\right)_{u \in \mathbb{Z}}$ an independent copy of $\left(\varepsilon_{u}\right)_{u \in \mathbb{Z}}$. Define $U_{n}\left(h,\left(\varepsilon_{u}\right)_{u \in \mathbb{Z}}\right):=\sum_{1 \leq i<j \leq n} h\left(V_{i}, V_{j}\right)-\mathbb{E}\left[h\left(V_{i}, V_{j}\right)\right]=$ $\sum_{1 \leq i<j \leq n} H_{i, j}$, where $V_{j}=\left(\varepsilon_{u}\right)_{u=j-\ell}^{j+\ell}, V_{j}^{\prime}=\left(\varepsilon_{u}^{\prime}\right)_{u=j-\ell}^{j+\ell}$ and $H_{i, j}:=h\left(V_{i}, V_{j}\right)-$ $\mathbb{E}\left[h\left(V_{i}, V_{j}\right)\right]$. 
Then

$$
\begin{aligned}
& U_{n}\left(h,\left(\varepsilon_{u}\right)_{u \in \mathbb{Z}}\right) \\
& =(4 \ell+2)\left\lfloor\frac{n}{4 \ell+2}\right\rfloor{ }_{k=1}^{(4 \ell+2)\left(\left\lfloor\frac{n}{4 \ell+2}\right\rfloor\right)+1}\left(\mathbb{E}\left[h\left(V_{k}, V_{0}^{\prime}\right) \mid V_{k}\right]-\mathbb{E}\left[h\left(V_{k}, V_{0}^{\prime}\right)\right]\right) \\
& +\sum_{a, b \in[4 \ell+2]} U_{\left\lfloor\frac{n}{4 \ell+2}\right\rfloor}^{\text {ind }}\left(h_{a, b}^{(2)},\left(\varepsilon_{i}^{a, b}\right)\right)+\sum_{k=1}^{6} R_{n, k},
\end{aligned}
$$

where the function $h_{a, b}: \mathbb{R}^{4 \ell+2} \times \mathbb{R}^{4 \ell+2} \rightarrow \mathbb{R}$ is defined by

$$
\begin{aligned}
h_{a, b}^{(2)} & :=h_{a, b}\left(\left(x_{i}\right)_{i=1}^{4 \ell+2},\left(y_{j}\right)_{j=1}^{4 \ell+2}\right) \\
& -h_{a, b}\left(\left(x_{i}\right)_{i=1}^{4 \ell+2}, \varepsilon_{0}^{a, b}\right)-h_{a, b}\left(\left(y_{j}\right)_{j=1}^{4 \ell+2}, \varepsilon_{0}^{a, b}\right)+\mathbb{E}\left[h_{a, b}\left(\varepsilon_{0}^{a, b}, \varepsilon_{0}^{\prime a, b}\right)\right]
\end{aligned}
$$

and

- the function $h_{a, b}: \mathbb{R}^{4 \ell+2} \times \mathbb{R}^{4 \ell+2} \rightarrow \mathbb{R}$ is defined for $0 \leq a-b \leq 2 \ell$ by

$$
\begin{aligned}
& h_{a, b}\left(\left(x_{i}\right)_{i=1}^{4 \ell+2},\left(y_{j}\right)_{j=1}^{4 \ell+2}\right):=h\left(\left(x_{i+a-b}\right)_{i=1}^{2 \ell+1},\left(y_{j}\right)_{j=1}^{2 \ell+1}\right)+h\left(\left(y_{j+a-b}\right)_{j=1}^{2 \ell+1},\left(x_{i}\right)_{i=1}^{2 \ell+1}\right) \\
& \quad \text { and for }(2 \ell+1) \leq a-b \leq(4 \ell+2)-1 \text { by } \\
& h_{a, b}\left(\left(x_{i}\right)_{i=1}^{4 \ell+2},\left(y_{j}\right)_{j=1}^{4 \ell+2}\right) \\
& \quad:=h\left(\left(x_{i+a-b-(2 \ell+1)}\right)_{i=1}^{2 \ell+1},\left(y_{j}\right)_{j=1}^{2 \ell+1}\right)+h\left(\left(y_{j+a-b-(2 \ell+1)}\right)_{j=1}^{2 \ell+1},\left(x_{i}\right)_{i=1}^{2 \ell+1}\right),
\end{aligned}
$$

- the random vectors $\varepsilon_{u}^{a, b}$ are defined by

$$
\begin{gathered}
\varepsilon_{u}^{a, b}:=\left(\varepsilon_{j}\right)_{j=u(4 \ell+2)+b-\ell}^{(u+1)(4 \ell+2)+b-\ell-1}, 0 \leq a-b \leq 2 \ell ; \\
\varepsilon_{u}^{a, b}:=\left(\varepsilon_{j}\right)_{j=u(4 \ell+2)+b+\ell+1}^{(u+1)(4 \ell+2)+b+\ell},(2 \ell+1) \leq a-b \leq(4 \ell+2)-1 ;
\end{gathered}
$$

- the remainder terms are defined (with the convention that $\sum_{u=1}^{-k}=0, k \leq 0$ ) by

$$
\begin{aligned}
& R_{n, 1,1}:=\sum_{j=(4 \ell+2)\left\lfloor\frac{n}{4 \ell+2}\right\rfloor+1}^{n} \sum_{i=(4 \ell+2)\left\lfloor\frac{j-1}{4 \ell+2}\right\rfloor+1}^{j-1} H_{i, j} \\
& R_{n, 1,2}:=\sum_{a=1}^{4 \ell+2} \sum_{j=(4 \ell+2)\left\lfloor\frac{n}{4 \ell+2}\right\rfloor+1}^{n} \sum_{k=0}^{\left\lfloor\frac{j-1}{4 \ell+2}\right\rfloor-1} H_{(4 \ell+2) k+a, j} \text {; } \\
& R_{n, 2}:=\sum_{u=0}^{\left\lfloor\frac{n}{4 \ell+2}\right\rfloor} \sum_{\substack{a, b \in[4 \ell+2] \\
a<b}} H_{u(4 \ell+2)+a, u(4 \ell+2)+b} \\
& R_{n, 3}:=\sum_{v=1}^{\left\lfloor\frac{n}{4 \ell+2}\right\rfloor-1} \sum_{\substack{a, b \in[4 \ell+2] \\
0 \leq a-b \leq(2 \ell+1)-1}}\left(H_{a, v(4 \ell+2)+b}+H_{b, v(4 \ell+2)+a}\right)
\end{aligned}
$$




$$
\begin{gathered}
R_{n, 4}=\sum_{\substack{a, b \in[4 \ell+2] \\
(2 \ell+1) \leq a-b \leq(4 \ell+2)-1}} \sum_{v=1}^{\left\lfloor\frac{n}{4 \ell+2}\right\rfloor-1}\left(H_{a,(v+1)(4 \ell+2)+b}+H_{(4 \ell+2)+b, v(4 \ell+2)+a}\right) \\
R_{n, 5}=\sum_{\substack{a, b \in[4 \ell+2] \\
(2 \ell+1) \leq a-b \leq(4 \ell+2)-1}} \sum_{\substack{\left\lfloor\frac{n}{4 \ell+2}\right\rfloor \\
R_{n, 6}}}=\sum_{\substack{a, b \in[4 \ell+2] \\
(2 \ell+1) \leq a-b \leq(4 \ell+2)-1}} \sum_{v=1}^{\left\lfloor\frac{n}{4 \ell+2}\right\rfloor-1}\left(H_{u(4 \ell+2)+a,(u+1)(4 \ell+2)+b}-H_{u(4 \ell+2)+a, 2 m(2 \ell+1)+b}\right) \\
\left.R_{b, v(4 \ell+2)+a}-H_{v(4 \ell+2)+b, v(4 \ell+2)+a}\right) .
\end{gathered}
$$

To sum up, the centered $U$-statistic whose data is a function of $2 \ell+1$ i.i.d. random variables can be decomposed as a partial sum of a strictly stationary sequence, a sum of degenerated $U$-statistics plus a remainder term.

Proof of Proposition 1.1: By the assumption (1.18), the following equality holds almost surely:

$$
\begin{aligned}
U_{n}\left(h, f,\left(\varepsilon_{u}\right)_{u \in \mathbb{Z}}\right)= & U_{n}\left(h, f_{0},\left(\varepsilon_{u}\right)_{u \in \mathbb{Z}}\right) \\
& +\sum_{\ell=1}^{+\infty}\left(U_{n}\left(h, f_{\ell},\left(\varepsilon_{u}\right)_{u \in \mathbb{Z}}\right)-U_{n}\left(h, f_{\ell-1},\left(\varepsilon_{u}\right)_{u \in \mathbb{Z}}\right)\right) .
\end{aligned}
$$

Since $U_{n}\left(h, f_{0},\left(\varepsilon_{u}\right)_{u \in \mathbb{Z}}\right)$ is a $U$-statistic of independent identically distributed data, it can be treated by the classical Hoeffding's decomposition written in (1.15).

To proceed, we need to decompose for a fixed $\ell \geq 1$ the term $U_{n}\left(h, f_{\ell},\left(\varepsilon_{u}\right)_{u \in \mathbb{Z}}\right)-$ $U_{n}\left(h, f_{\ell-1},\left(\varepsilon_{u}\right)_{u \in \mathbb{Z}}\right)$. To this aim, we apply Lemma 2.2 in the following setting: the function $h$ is replaced by $\widetilde{h}: \mathbb{R}^{2 \ell+1} \times \mathbb{R}^{2 \ell+1} \rightarrow \mathbb{R}$, which is defined by

$$
\begin{aligned}
& \widetilde{h}\left(\left(x_{i}\right)_{i=1}^{2 \ell+1},\left(y_{j}\right)_{j=1}^{2 \ell+1}\right) \\
= & h\left(f_{\ell}\left(\left(x_{i}\right)_{i=1}^{2 \ell+1}\right), f_{\ell}\left(\left(y_{j}\right)_{j=1}^{2 \ell+1}\right)\right)-h\left(f_{\ell-1}\left(\left(x_{i}\right)_{i=2}^{2 \ell}\right), f_{\ell-1}\left(\left(y_{j}\right)_{j=2}^{2 \ell}\right)\right) .
\end{aligned}
$$

In this way,

$U_{n}\left(h, f_{\ell},\left(\varepsilon_{u}\right)_{u \in \mathbb{Z}}\right)-U_{n}\left(h, f_{\ell-1},\left(\varepsilon_{u}\right)_{u \in \mathbb{Z}}\right)=\sum_{1 \leq i<j \leq n} \tilde{h}\left(\left(\varepsilon_{i-u}\right)_{u=-\ell}^{\ell},\left(\varepsilon_{j-v}\right)_{v=-\ell}^{\ell}\right)$

and with the notation $V_{i, \ell}=\left(\varepsilon_{u}\right)_{u=i-\ell}^{i+\ell}$, Lemma 2.1 gives

$$
\begin{aligned}
& U_{n}\left(h, f_{\ell},\left(\varepsilon_{u}\right)_{u \in \mathbb{Z}}\right)-U_{n}\left(h, f_{\ell-1},\left(\varepsilon_{u}\right)_{u \in \mathbb{Z}}\right) \\
& -\mathbb{E}\left[U_{n}\left(h, f_{\ell},\left(\varepsilon_{u}\right)_{u \in \mathbb{Z}}\right)-U_{n}\left(h, f_{\ell-1},\left(\varepsilon_{u}\right)_{u \in \mathbb{Z}}\right)\right] \\
& =(4 \ell+2)\left\lfloor\frac{n}{4 \ell+2}\right\rfloor \sum_{k=1}^{(4 \ell+2)\left\lfloor\frac{n}{4 \ell+2}\right\rfloor+1}\left(\mathbb{E}\left[\widetilde{h}\left(V_{k, \ell}, V_{0, \ell}^{\prime}\right) \mid V_{k, \ell}\right]-\mathbb{E}\left[\widetilde{h}\left(V_{k, \ell}, V_{0, \ell}^{\prime}\right) \mid V_{k, \ell}\right]\right) \\
& +\sum_{a, b \in[4 \ell+2]} U_{\left\lfloor\frac{n}{4 \ell+2}\right\rfloor}^{\text {ind }}\left(\widetilde{h}_{a, b}^{(2)},\left(\varepsilon_{i}^{a, b}\right)\right)+\sum_{k=1}^{6} R_{n, k} .
\end{aligned}
$$


Observe that

$$
\begin{aligned}
\mathbb{E}\left[\widetilde{h}\left(V_{k, \ell}, V_{0, \ell}^{\prime}\right) \mid V_{k, \ell}\right]=\mathbb{E}[ & \left.h\left(f_{\ell}\left(V_{k, \ell}\right), f_{\ell}\left(V_{0, \ell}^{\prime}\right)\right) \mid V_{k, \ell}\right] \\
& -\mathbb{E}\left[h\left(f_{\ell-1}\left(V_{k, \ell-1}\right), f_{\ell-1}\left(V_{0, \ell-1}^{\prime}\right) \mid V_{k, \ell}\right)\right]
\end{aligned}
$$

and using Lemma A.5, this equality becomes

$$
\begin{aligned}
\mathbb{E}\left[\widetilde{h}\left(V_{k, \ell}, V_{0, \ell}^{\prime}\right) \mid V_{k, \ell}\right]=\mathbb{E}[ & {\left[h\left(f_{\ell}\left(V_{k, \ell}\right), f_{\ell}\left(V_{0, \ell}^{\prime}\right)\right) \mid V_{k, \ell}\right] } \\
& -\mathbb{E}\left[h\left(f_{\ell-1}\left(V_{k, \ell-1}\right), f_{\ell-1}\left(V_{0, \ell-1}^{\prime}\right)\right) \mid V_{k, \ell-1}\right] .
\end{aligned}
$$

Moreover,

$$
U_{\left\lfloor\frac{n}{4 \ell+2}\right\rfloor}^{\text {ind }}\left(\widetilde{h}_{a, b}^{(2)},\left(\varepsilon_{i}^{a, b}\right)\right)=\sum_{1 \leq u<v \leq\left\lfloor\frac{n}{4 \ell+2}\right\rfloor}\left(Y_{\ell, u, v}^{a, b}+Y_{\ell, u, v}^{b, a}-Y_{\ell-1, u, v}^{a, b}-Y_{\ell-1, u, v}^{b, a}\right)
$$

where

$$
\begin{aligned}
& Y_{\ell, u, v}^{a, b}=h\left(f_{\ell}\left(V_{(4 \ell+2) u+a, \ell}\right), f_{\ell}\left(V_{(4 \ell+2) v+b, \ell}\right)\right)- \\
& \mathbb{E}\left[h\left(f_{\ell}\left(V_{(4 \ell+2) u+a, \ell}\right), f_{\ell}\left(V_{0, \ell}^{\prime}\right)\right) \mid V_{(4 \ell+2) u+a, \ell}\right] \\
& -\mathbb{E}\left[h\left(f_{\ell}\left(V_{(4 \ell+2) v+b, \ell}\right), f_{\ell}\left(V_{0, \ell}^{\prime}\right)\right) \mid V_{(4 \ell+2) v+b, \ell}\right]+\mathbb{E}\left[h\left(f_{\ell}\left(V_{0, \ell}\right), f_{\ell}\left(V_{0, \ell}^{\prime}\right)\right)\right] .
\end{aligned}
$$

We conclude by collecting all the terms.

\subsection{Proof of the results of Subsection 1.4.}

Proof of Proposition 1.2: It will be more convenient to work with dyadics, since the martingale property will be useful to handle the maximums. First observe that $M_{p} \leq 2^{1 / p} M_{p}^{\prime}$, where

$$
M_{p}^{\prime}:=\sup _{N \geq 1} 2^{-2 N / p} \max _{2 \leq n \leq 2^{N}}\left|U_{n}^{\text {ind }}\left(h,\left(\varepsilon_{i}\right)_{i \in \mathbb{Z}}\right)-\mathbb{E}\left[U_{n}^{\text {ind }}\left(h,\left(\varepsilon_{i}\right)_{i \in \mathbb{Z}}\right)\right]\right| .
$$

For a fixed integer $n$, consider the event

$$
A_{N}:=\left\{2^{-2 N / p} \max _{2 \leq n \leq 2^{N}}\left|U_{n}^{\text {ind }}\left(h,\left(\varepsilon_{i}\right)_{i \in \mathbb{Z}}\right)\right|>2\right\} .
$$

It suffices to prove that there exists a constant $c_{p}$ (depending only on $p$ ) such that

$$
\sum_{N=1}^{+\infty} \mathbb{P}\left(A_{N}\right) \leq c_{p} \mathbb{E}\left[\left|h\left(\varepsilon_{0}, \varepsilon_{1}\right)\right|^{p}\right]
$$

Indeed, item 1 follows from an application of (2.32) to $h / \varepsilon$ for a positive $\varepsilon$ and the Borel-Cantelli lemma. In order to prove item 2, we notice that $\mathbb{P}\left\{M_{p}^{\prime}>2\right\} \leq$ $\sum_{N=1}^{+\infty} \mathbb{P}\left(A_{N}\right)$ and we apply (2.32) to $h / x$ for each positive $x$. Consequently, we focus on establishing a satisfactory bound for $\mathbb{P}\left(A_{N}\right)$.

Define for $j \geq 2$ the random variable $D_{j}:=\sum_{i=1}^{j-1} h\left(\varepsilon_{i}, \varepsilon_{j}\right)$. Let $\mathcal{F}_{j}$ denote the $\sigma$-algebra generated by the random variables $\varepsilon_{k}, 1 \leq k \leq j$. Define

$$
\begin{gathered}
D_{j}^{\prime}:=D_{j} \mathbf{1}\left\{\left|D_{j}\right| \leq 2^{2 N / p}\right\}-\mathbb{E}\left[D_{j} \mathbf{1}\left\{\left|D_{j}\right| \leq 2^{2 N / p}\right\} \mid \mathcal{F}_{j-1}\right] \text { and } \\
D_{j}^{\prime \prime}:=D_{j} \mathbf{1}\left\{\left|D_{j}\right|>2^{2 N / p}\right\}-\mathbb{E}\left[D_{j} \mathbf{1}\left\{\left|D_{j}\right|>2^{2 N / p}\right\} \mid \mathcal{F}_{j-1}\right] .
\end{gathered}
$$


Since $\mathbb{E}\left[D_{j} \mid \mathcal{F}_{j-1}\right]=0$, it follows that $D_{j}=D_{j}^{\prime}+D_{j}^{\prime \prime}$ hence $A_{N} \subset A_{N}^{\prime} \cup A_{N}^{\prime \prime}$, where

$$
\begin{gathered}
A_{N}^{\prime}:=\left\{2^{-4 N / p} \max _{2 \leq k \leq 2^{N}}\left|\sum_{j=2}^{k} D_{j}^{\prime}\right|>1\right\} \text { and } \\
A_{N}^{\prime \prime}:=\left\{2^{-4 n / p} \max _{2 \leq k \leq 2^{N}}\left|\sum_{j=2}^{k} D_{j}^{\prime \prime}\right|>1\right\} .
\end{gathered}
$$

Let us bound $p_{N}^{\prime}:=\mathbb{P}\left(A_{N}^{\prime}\right)$. Markov's inequality entails

$$
p_{N}^{\prime} \leq 2^{-4 N / p} \mathbb{E}\left[\max _{2 \leq k \leq 2^{N}}\left|\sum_{j=2}^{k} D_{j}^{\prime}\right|^{2}\right]
$$

and since $\left(D_{j}^{\prime}\right)_{j \geq 2}$ is a martingale differences sequence, we obtain by Doob's inequality and orthogonality of increments that

$$
p_{N}^{\prime} \leq 2^{1-4 N / p} \sum_{j=2}^{2^{N}} \mathbb{E}\left[D_{j}^{\prime 2}\right] \leq 2^{2-4 N / p} \sum_{j=2}^{2^{N}} \mathbb{E}\left[D_{j}^{2} \mathbf{1}\left\{\left|D_{j}\right| \leq 2^{N / p}\right\}\right] .
$$

Now, we use

$$
\begin{aligned}
\mathbb{E}\left[D_{j}^{2} \mathbf{1}\left\{\left|D_{j}\right| \leq 2^{2 N / p}\right\}\right] & =2 \int_{0}^{2^{2 N / p}} t \mathbb{P}\left\{t<\left|D_{j}\right| \leq 2^{2 N / p}\right\} \mathrm{d} t \\
& =2 \int_{0}^{2^{2 N / p}} t \mathbb{P}\left\{\left|D_{j}\right|>t\right\} \mathrm{d} t-2^{2 N / p} \mathbb{P}\left\{\left|D_{j}\right|>2^{2 N / p}\right\} \\
& \leq 2 \int_{0}^{2^{2 N / p}} t \mathbb{P}\left\{\left|D_{j}\right|>t\right\} \mathrm{d} t
\end{aligned}
$$

and after the substitution $s=2^{-2 N / p} t$, we get

$$
\mathbb{E}\left[D_{j}^{2} \mathbf{1}\left\{\left|D_{j}\right| \leq 2^{2 N / p}\right\}\right] \leq 2^{1+4 N / p} \int_{0}^{1} s \mathbb{P}\left\{\left|D_{j}\right|>2^{2 N / p} s\right\} \mathrm{d} s .
$$

We thus obtained the estimate

$$
p_{N}^{\prime} \leq 8 \sum_{j=2}^{2^{N}} \int_{0}^{1} s \mathbb{P}\left\{\left|D_{j}\right|>2^{2 N / p} s\right\} \mathrm{d} s .
$$

In order to bound $p_{N}^{\prime \prime}:=\mathbb{P}\left(A_{N}^{\prime \prime}\right)$, we start by Markov's inequality to get

$$
p_{N}^{\prime \prime} \leq 2^{-2 N / p} \sum_{j=2}^{2^{N}} \mathbb{E}\left[\left|D_{j}^{\prime \prime}\right|\right] \leq 2^{1-2 N / p} \sum_{j=2}^{2^{N}} \mathbb{E}\left[\left|D_{j}\right| \mathbf{1}\left\{\left|D_{j}\right|>2^{2 N / p}\right\}\right] .
$$

Since there exists a constant $C_{p}$ depending only on $p$ such that

$$
\begin{aligned}
\mathbb{E}\left[\left|D_{j}\right| \mathbf{1}\left\{\left|D_{j}\right|>2^{2 N / p}\right\}\right]=2^{2 N / p} \mathbb{P}\left\{\left|D_{j}\right|>2^{2 N / p}\right\}+\int_{2^{2 N / p}}^{+\infty} \mathbb{P}\left\{\left|D_{j}\right|>t\right\} \mathrm{d} t \\
\leq C_{p} \int_{2^{2 N / p-1}}^{+\infty} \mathbb{P}\left\{\left|D_{j}\right|>t\right\} \mathrm{d} t,
\end{aligned}
$$


we derive after the substitution $t=2^{2 N / p} s$ that

$$
p_{N}^{\prime \prime} \leq C_{p} 2 \sum_{j=2}^{2^{N}} \int_{1 / 2}^{+\infty} \mathbb{P}\left\{\left|D_{j}\right|>2^{2 N / p} s\right\} \mathrm{d} s .
$$

The combination of (2.42) with (2.45) yields

$$
\mathbb{P}\left(A_{N}\right) \leq C_{p} \sum_{j=2}^{2^{N}} \int_{0}^{+\infty} \min \{1, s\} \mathbb{P}\left\{\left|D_{j}\right|>2^{2 N / p} s\right\} \mathrm{d} s .
$$

We are thus reduced to control the tail of $D_{j}$, which will be done by using Proposition A.1. Our particular setting permits some simplification of the involved terms.

We first observe that $D_{j}$ has the same distribution as $\sum_{i=1}^{j-1} h\left(\varepsilon_{0}, \varepsilon_{i}\right)$ (since the vectors $\left(\varepsilon_{1}, \ldots, \varepsilon_{j-1}, \varepsilon_{j}\right)$ and $\left(\varepsilon_{1}, \ldots, \varepsilon_{j-1}, \varepsilon_{0}\right)$ are identically distributed). Define $d_{i}:=h\left(\varepsilon_{0}, \varepsilon_{i}\right)$. Since $\mathbb{E}\left[h\left(\varepsilon_{0}, x\right)\right]=0$ for all $x \in \mathbb{R}^{d}$, the sequence $\left(d_{i}\right)_{i=1}^{d}$ is a martingale differences sequence for the filtration $\left(\mathcal{G}_{i}\right)_{i=1}^{n}$ where $\mathcal{G}_{i}$ is the $\sigma$-algebra generated by $\varepsilon_{k}, 0 \leq k \leq i$. We apply Proposition A.1 to $x=2^{2 n / p} s$ for a fixed positive $s$ and $q=2 p$. let $i \in\{1, \ldots, j-1\}$. By Lemma A.5 applied to $Y=d_{i}$, $\mathcal{F}=\sigma\left(\varepsilon_{0}\right)$ and $\mathcal{G}=\sigma\left(\varepsilon_{1}, \ldots, \varepsilon_{i-1}\right)$, we have

$$
\mathbb{E}\left[\left|d_{i}\right|^{p} \mid \mathcal{G}_{i-1}\right]=\mathbb{E}\left[\left|h\left(\varepsilon_{0}, \varepsilon_{j}\right)\right|^{p} \mid \sigma\left(\varepsilon_{0}\right)\right] .
$$

Using Lemma A.6 with $Y=\varepsilon_{j}, Z=\varepsilon_{0}$ and $f=h$, we derive that

$$
\mathbb{E}\left[\left|d_{i}\right|^{p} \mid \mathcal{G}_{i-1}\right]=\mathbb{E}\left[\left|h\left(\varepsilon_{0}, \varepsilon_{1}\right)\right|^{p} \mid \sigma\left(\varepsilon_{0}\right)\right] .
$$

Using this equality combined with the fact that the random variables $d_{i}, 1 \leq i \leq$ $j-1$ have the same distribution as $d_{1}$, one gets

$$
\begin{aligned}
& \mathbb{P}\left\{\left|D_{j}\right|>2^{2 N / p} s\right\} \leq c_{1}(j-1) \int_{0}^{1} \mathbb{P}\left\{\left|d_{1}\right|>x 2^{2 N / p} u s c_{2}\right\} u^{q-1} \mathrm{~d} u \\
& +c_{1} \int_{0}^{1} \mathbb{P}\left\{(j-1)^{1 / p}\left(\mathbb{E}\left[\left|h\left(\varepsilon_{0}, \varepsilon_{1}\right)\right|^{p} \mid \sigma\left(\varepsilon_{0}\right)\right]\right)^{1 / p}>2^{2 N / p} s u c_{2}\right\} u^{q-1} \mathrm{~d} u .
\end{aligned}
$$

In view of (2.46), we derive that

$$
\begin{aligned}
& \mathbb{P}\left(A_{N}\right) \leq c_{1} 2^{2 N} \int_{0}^{+\infty} \int_{0}^{1} \mathbb{P}\left\{\left|d_{1}\right|>x 2^{2 N / p} s u c_{2}\right\} u^{2 p-1} \mathrm{~d} u \min \{1, s\} \mathrm{d} s \\
& +c_{1} 2^{N} \int_{0}^{+\infty} \int_{0}^{1} \mathbb{P}\left\{\left(\mathbb{E}\left[\left|h\left(\varepsilon_{0}, \varepsilon_{1}\right)\right|^{p} \mid \sigma\left(\varepsilon_{0}\right)\right]\right)^{1 / p}>2^{N / p} \text { suc }_{2}\right\} u^{2 p-1} \mathrm{~d} u \min \{1, s\} \mathrm{d} s .
\end{aligned}
$$

Summing over $N$, we get (2.32) in view of the inequality

$$
\sum_{N \geq 1} 2^{2 N} \mathbb{P}\left(Y>2^{2 N / p}\right) \leq 2 \mathbb{E}\left[Y^{p}\right]
$$

for a non-negative random variable $Y$ and the convergence of the integrals $\int_{0}^{1} u^{p-1} \mathrm{~d} u$ and $\int_{0}^{+\infty} \min \{1, s\} s^{-p} \mathrm{~d} s$. This ends the proof of Proposition 1.2. 
2.2.1. Treatment of $R_{n, 1,1}$ and $R_{n, 1,2}$. Recall that

$$
\begin{aligned}
& H_{i, j}^{(\ell)}:=h\left(f_{\ell}\left(V_{i, \ell}\right), f_{\ell}\left(V_{j, \ell}\right)\right)-\mathbb{E}\left[h\left(f_{\ell}\left(V_{i, \ell}\right), f_{\ell}\left(V_{j, \ell}\right)\right)\right] \\
& \quad-\left(h\left(f_{\ell-1}\left(V_{i, \ell-1}\right), f_{\ell-1}\left(V_{j, \ell-1}\right)\right)-\mathbb{E}\left[h\left(f_{\ell-1}\left(V_{i, \ell-1}\right), f_{\ell-1}\left(V_{j, \ell-1}\right)\right)\right]\right) .
\end{aligned}
$$

and

$$
\begin{gathered}
R_{n, 1,1}:=\sum_{\ell \geq 1} Y_{n, \ell} ; \quad Y_{n, \ell}:=\sum_{j=(4 \ell+2)\left\lfloor\frac{n}{\lfloor\ell+2}\right\rfloor+1}^{n} \sum_{i=(4 \ell+2)\left\lfloor\frac{j-1}{4 \ell+2}\right\rfloor+1}^{j-1} H_{i, j}^{(\ell)} \\
R_{n, 1,2}:=\sum_{\ell \geq 1} Z_{n, \ell} ; \quad Z_{n, \ell}:=\sum_{a=1}^{4 \ell+2} \sum_{j=(4 \ell+2)\left\lfloor\frac{n}{4 \ell+2}\right\rfloor+1}^{n} \sum_{k=0}^{\left\lfloor\frac{j-1}{4 \ell+2}\right\rfloor-1} H_{(4 \ell+2) k+a, j}^{(\ell)} .
\end{gathered}
$$

For a fix $\ell \geq 1$, we evaluate the contribution of of $Y_{n, \ell}$ and $Z_{n, \ell}$.

Lemma 2.3. Let $\ell \geq 1$. The following inequalities hold:

$$
\begin{aligned}
& \left\|\sup _{n \geq 1} \frac{1}{n^{1+1 / p}}\left|Y_{n, \ell}\right|\right\|_{p, \infty} \leq c_{p} \ell^{1-1 / p} \theta_{\ell, p} \\
& \left\|\sup _{n \geq 1} \frac{1}{n^{1+1 / p}}\left|Z_{n, \ell}\right|\right\|_{p, \infty} \leq c_{p} \ell^{1-1 / p} \theta_{\ell, p},
\end{aligned}
$$

where $c_{p}$ depends only on $p$.

Proof: First observe that $Y_{n, \ell}$ is a sum of at most $(4 \ell+2)^{2}$ random variables whose weak- $\mathbb{L}^{p}$-norm does not exceed $\theta_{\ell, p}$ hence by cutting the supremum where $n$ is between two consecutive multiples of $4 \ell+2$ gives

$$
\begin{aligned}
\left\|\sup _{n \geq 1} \frac{1}{n^{1+1 / p}}\left|Y_{n, \ell}\right|\right\|_{p, \infty} & \leq\left(\sum_{n \geq 1}\left\|\frac{1}{n^{1+1 / p}}\left|Y_{n, \ell}\right|\right\|_{p, \infty}^{p}\right)^{1 / p} \\
& \leq(4 \ell+2)^{2-1-1 / p}\left(\sum_{n \geq 1} n^{-1-p}\right)^{1 / p} \theta_{\ell, p}
\end{aligned}
$$

In order to treat $Z_{n, \ell}$, we decompose it as $Z_{n, \ell}^{\prime}+Z_{n, \ell}^{\prime \prime}$, where

$$
\begin{gathered}
Z_{n, \ell}^{\prime}=\sum_{a=1}^{4 \ell+2} \sum_{j=(4 \ell+2)\left\lfloor\frac{n}{\lfloor\ell+2}\right\rfloor+1}^{n} \sum_{k=0}^{\left\lfloor\frac{j-1}{4 \ell+2}\right\rfloor-1}\left(H_{(4 \ell+2) k+a, j}^{(\ell)}-\mathbb{E}\left[H_{(4 \ell+2) k+a, j}^{(\ell)} \mid V_{j, \ell}\right]\right) \\
Z_{n, \ell}^{\prime \prime}=\sum_{a=1}^{4 \ell+2} \sum_{j=(4 \ell+2)\left\lfloor\frac{n}{4 \ell+2}\right\rfloor+1}^{n} \sum_{k=0}^{\left\lfloor\frac{j-1}{4 \ell+2}\right\rfloor-1} \mathbb{E}\left[H_{(4 \ell+2) k+a, j}^{(\ell)} \mid V_{j, \ell}\right]
\end{gathered}
$$


Using (1.25), it follows that

$$
\begin{aligned}
& \left\|\sup _{n \geq 1} \frac{1}{n^{1+1 / p}}\left|Z_{n, \ell}^{\prime}\right|\right\|_{p, \infty} \leq\left(\sum_{n \geq 1}\left\|\frac{1}{n^{1+1 / p}}\left|Z_{n, \ell}^{\prime}\right|\right\|_{p, \infty}^{p}\right)^{1 / p} \\
& \leq\left(\sum_{N \geq 1} N^{-p-1}(4 \ell+2)^{-p-1}\left(\sum_{n=(4 \ell+2) N}^{(4 \ell+2)(N+1)-1}\left\|Z_{n, \ell}^{\prime}\right\|_{p, \infty}\right)^{p}\right)^{1 / p}
\end{aligned}
$$

and for all $n$ such that $(4 \ell+2) N \leq n \leq(4 \ell+2)(N+1)-1$,

$$
\begin{aligned}
& \left\|Z_{n, \ell}^{\prime}\right\|_{p, \infty} \\
& \leq \sum_{a=1}^{4 \ell+2} \sum_{j=(4 \ell+2)\left\lfloor\frac{n}{4 \ell+2}\right\rfloor+1}^{n}\left\|\sum_{k=0}^{\left\lfloor\frac{n}{4 \ell+2}\right\rfloor-1}\left(H_{(4 \ell+2) k+a, j}^{(\ell)}-\mathbb{E}\left[H_{(4 \ell+2) k+a, j}^{(\ell)} \mid V_{j, \ell}\right]\right)\right\|_{p, \infty} \\
& \leq \sum_{a=1}^{4 \ell+2} \sum_{j=(4 \ell+2) N+1}^{(4 \ell+2)(N+1)}\left\|\sum_{k=0}^{N-1}\left(H_{(4 \ell+2) k+a, j}^{(\ell)}-\mathbb{E}\left[H_{(4 \ell+2) k+a, j}^{(\ell)} \mid V_{j, \ell}\right]\right)\right\|_{p, \infty}
\end{aligned}
$$

hence

$$
\begin{aligned}
& \left\|\sup _{n \geq 1} \frac{1}{n^{1+1 / p}}\left|Z_{n, \ell}^{\prime}\right|\right\|_{p, \infty}^{p} \\
& \leq \sum_{N \geq 1} N^{-p-1}(4 \ell+2)^{-p-1}\left(\sum_{a=1}^{4 \ell+2} \sum_{j=(4 \ell+2) N+1}^{(4 \ell+2)(N+1)}\right. \\
& \left.\left\|\sum_{k=0}^{N-1}\left(H_{(4 \ell+2) k+a, j}^{(\ell)}-\mathbb{E}\left[H_{(4 \ell+2) k+a, j}^{(\ell)} \mid V_{j, \ell}\right]\right)\right\|_{p, \infty}\right)^{p} .
\end{aligned}
$$

For all fixed $j$, we notice using Lemma A.5 that

$$
\left(H_{(4 \ell+2) k+a, j}^{(\ell)}-\mathbb{E}\left[H_{(4 \ell+2) k+a, j}^{(\ell)} \mid V_{j, \ell}\right]\right)_{0 \leq k \leq N-1}
$$

is a martingale differences sequence with respect to the filtration $\left(\mathcal{F}_{k}\right)_{0 \leq k \leq N-1}$ where

$$
\mathcal{F}_{k}:=\sigma\left(V_{j, \ell}\right) \vee \sigma\left(V_{(4 \ell+2) i+a, j}, i \leq k\right)
$$

hence by Burkholder's inequality,

$$
\begin{gathered}
\left\|\sum_{k=0}^{N-1}\left(H_{(4 \ell+2) k+a, j}^{(\ell)}-\mathbb{E}\left[H_{(4 \ell+2) k+a, j}^{(\ell)} \mid V_{j, \ell}\right]\right)\right\|_{p, \infty}^{p} \\
\leq c_{p} \sum_{k=0}^{N-1}\left\|H_{(4 \ell+2) k+a, j}^{(\ell)}-\mathbb{E}\left[H_{(4 \ell+2) k+a, j}^{(\ell)} \mid V_{j, \ell}\right]\right\|_{p, \infty}^{p} \\
\leq 2^{p} c_{p} \sum_{k=0}^{N-1}\left\|H_{(4 \ell+2) k+a, j}^{(\ell)}\right\|_{p}^{p} \leq 2^{p} c_{p} N \theta_{\ell, p}^{p}
\end{gathered}
$$


and plugging this estimate into (2.58) gives

$$
\begin{aligned}
& \left\|\sup _{n \geq 1} \frac{1}{n^{1+1 / p}}\left|Z_{n, \ell}^{\prime}\right|\right\|_{p, \infty} \\
& \leq C_{p}\left(\sum_{N \geq 1} N^{-p-1}(4 \ell+2)^{-p-1}(4 \ell+2)^{2 p}\left(N \theta_{\ell, p}^{p}\right)^{p}\right)^{1 / p} \\
& \leq C_{p}^{\prime} \theta_{\ell, p} \ell^{1-1 / p} .
\end{aligned}
$$

In order to treat the contribution of $Z_{n, \ell}^{\prime \prime}$, we observe that $\mathbb{E}\left[H_{(4 \ell+2) k+a, j}^{(\ell)} \mid V_{j, \ell}\right]$ is independent of $k$ hence

$$
Z_{n, \ell}^{\prime \prime}=\sum_{a=1}^{4 \ell+2} \sum_{j=(4 \ell+2)\left\lfloor\frac{n}{4 \ell+2}\right\rfloor+1}^{n}\left\lfloor\frac{n}{4 \ell+2}\right\rfloor \mathbb{E}\left[H_{a, j}^{(\ell)} \mid V_{j, \ell}\right]
$$

Consequently, the control of the contribution of $\sup _{n \geq 1} n^{-1-1 / p}\left|Z_{n, \ell}^{\prime \prime}\right|$ can be done thanks to Proposition A.2.

This ends the proof of Lemma 2.3.

2.2.2. Treatment of terms of the form $\sum_{u} H_{a,(4 \ell+2) u+b}^{(\ell)}$ and $\sum_{u} H_{(\ell \ell+2) u+a,(4 \ell+2)\left\lfloor\frac{n}{4 \ell+2}\right\rfloor+b}^{(\ell)}$.

Lemma 2.4. For all $\ell \geq 1$ and all $a, b \in[4 \ell+2]$, the following inequality holds

$$
\left\|\sup _{n \geq 1} \frac{1}{n^{1+1 / p}} \mid \sum_{u=1}^{\left\lfloor\frac{n}{4 \ell+2}\right\rfloor} H_{a,(4 \ell+2) u+b}^{(\ell)}\right\| \|_{p, \infty} \leq c_{p}(4 \ell+2)^{-1-1 / p} \theta_{\ell, p} .
$$

Lemma 2.5. For all $\ell \geq 1$ and all $a, b \in[4 \ell+2]$, the following inequality holds

$$
\left\|\sup _{n \geq 1} \frac{1}{n^{3 / 2}} \mid \sum_{u=1}^{\left\lfloor\frac{n}{4 \ell+2}\right\rfloor} H_{(4 \ell+2) u+a,(4 \ell+2)\left\lfloor\frac{n}{4 \ell+2}\right\rfloor+b}^{(\ell)}\right\| \|_{p, \infty} \leq c_{p}(4 \ell+2)^{-1-1 / p} \theta_{\ell, p} .
$$

These two lemmas are the consequence of the following observations.

(1) We first assume that $0 \leq a-b \leq 2 \ell$; if not we add and subtract $H_{a,(4 \ell+2)(u+1)+b}^{(\ell)}$ and use telescoping to treat instead $\sum_{u=1}^{\left\lfloor\frac{n}{4 \ell+2}\right\rfloor} H_{a,(4 \ell+2)(u+1)+b}^{(\ell)}$ (where we will apply the previous case to $\widetilde{a}=a$ and $\widetilde{b}=b+2 \ell+1)$. A similar method can be used to treat the sum involved in Lemma 2.5 .

(2) The supremum involved in the statement can be restricted to the integers $n$ with are a multiple of $4 \ell+2$.

(3) For all integer $N$ and all sequence of random variables $W_{i, j}, i, j \geq 1$, we write

$$
S_{n}:=\sum_{1 \leq i<j \leq n} W_{i, j}, \quad S_{n}^{\prime}:=\sum_{0 \leq i<j \leq n} W_{i, j}
$$


With the choice $W_{i, j}:=H_{(4 \ell+2) i+a,(4 \ell+2) j+b}^{(\ell)}$, the following equality holds:

$$
\sum_{u=1}^{\left\lfloor\frac{n}{4 \ell+2}\right\rfloor} H_{a,(4 \ell+2) u+b}^{(\ell)}=S_{N}^{\prime}-S_{N},
$$

when $n=(4 \ell+2) N$. Since $S_{N}^{\prime}$ and $S_{N}$ can be both expressed as $U$ statistics of i.i.d. data, we can use Corollary 1.3 to treat these terms.

Lemma 2.5 can be done in a similar way: we express this time the involved sum as $S_{N}-S_{N-1}$.

\subsubsection{Treatment of terms of the form $\sum_{u} H_{(4 \ell+2) u+a,(4 \ell+2) u+b}^{(\ell)}$.}

Lemma 2.6. For all $\ell \geq 1$ and all $a, b \in[4 \ell+2]$, the following inequality holds

$$
\left\|\sup _{n \geq 1} \frac{1}{n^{1+1 / p}}\left|\sum_{u=1}^{\left\lfloor\frac{n}{4 \ell+2}\right\rfloor} H_{(4 \ell+2) u+a,(4 \ell+2) u+b}^{(\ell)}\right|\right\| \|_{p, \infty} \leq c_{p}(4 \ell+2)^{-1-1 / p} \theta_{\ell, p} .
$$

This follows from the fact that $\left(H_{(4 \ell+2) u+a,(4 \ell+2) u+b}^{(\ell)}\right)_{u \geq 1}$ forms a two-dependent sequence.

2.2.4. Proof of Theorem 1.4 and Theorem 1.5. Theorem 1.4 is a consequence of the combination of Proposition 1.1, the estimates of Lemmas 2.3, 2.4, 2.6 and 2.5.

In order to prove Theorem 1.5, we start from the decomposition

$$
U_{n}\left(h, f,\left(\varepsilon_{i}\right)_{i \in \mathbb{Z}}\right)-\mathbb{E}\left[U_{n}\left(h, f,\left(\varepsilon_{i}\right)_{i \in \mathbb{Z}}\right)\right]=A_{n, L}+B_{n, L},
$$

where for a fixed $L, A_{n, L}$ is the sum for the indexes $\ell$ smaller or equal to $L$ (viewing the terms associated to $V_{k, 0}$ as the corresponding ones for $\ell=0$ ) and $B_{n, L}$ the remaining term. We have to prove that for each positive $\varepsilon$,

$$
\lim _{N \rightarrow+\infty} \mathbb{P}\left\{\sup _{n \geq N} \frac{1}{n^{1+1 / p}}\left|U_{n}\left(h, f,\left(\varepsilon_{i}\right)_{i \in \mathbb{Z}}\right)-\mathbb{E}\left[U_{n}\left(h, f,\left(\varepsilon_{i}\right)_{i \in \mathbb{Z}}\right)\right]\right|>2 \varepsilon\right\}=0 .
$$

Using (2.65) and the fact that $A_{n, L}$ consists of sums of terms which can be treated by Lemmas 2.3, 2.4, 2.6 and 2.5, we derive that for all fixed $L$,

$$
\begin{array}{r}
\limsup _{N \rightarrow+\infty} \mathbb{P}\left\{\sup _{n \geq N} \frac{1}{n^{1+1 / p}}\left|U_{n}\left(h, f,\left(\varepsilon_{i}\right)_{i \in \mathbb{Z}}\right)-\mathbb{E}\left[U_{n}\left(h, f,\left(\varepsilon_{i}\right)_{i \in \mathbb{Z}}\right)\right]\right|>2 \varepsilon\right\} \\
\leq \limsup _{N \rightarrow+\infty} \mathbb{P}\left\{\sup _{n \geq N} \frac{1}{n^{1+1 / p}}\left|B_{n, L}\right|>\varepsilon\right\} .
\end{array}
$$

Bounding the latter probability by $\varepsilon^{-p}\left\|\sup _{n \geq 1} \frac{1}{n^{1+1 / p}}\left|B_{n, L}\right|\right\|_{p, w}^{p}$ and using Lemmas 2.3, 2.4, 2.6 and 2.5, we can see that for some constant $C$ depending only on $p$,

$$
\left\|\sup _{n \geq 1} \frac{1}{n^{1+1 / p}}\left|B_{n, L}\right|\right\|_{p, w} \leq C \sum_{\ell \geq L} \ell^{2} \theta_{\ell, p}
$$

which can be made arbitrarily small.

2.3. Proof of Theorem 1.6. Like for the results on the strong law of larger numbers, we have to control the contribution of the extra-terms in the decomposition obtained in Proposition 1.1. 
2.3.1. Treatment of $R_{n, 1,1}$ and $R_{n, 1,2}$. Recall that

$$
\begin{aligned}
& H_{i, j}^{(\ell)}:=h\left(f_{\ell}\left(V_{i, \ell}\right), f_{\ell}\left(V_{j, \ell}\right)\right)-\mathbb{E}\left[h\left(f_{\ell}\left(V_{i, \ell}\right), f_{\ell}\left(V_{j, \ell}\right)\right)\right] \\
& \quad-\left(h\left(f_{\ell-1}\left(V_{i, \ell-1}\right), f_{\ell-1}\left(V_{j, \ell-1}\right)\right)-\mathbb{E}\left[h\left(f_{\ell-1}\left(V_{i, \ell-1}\right), f_{\ell-1}\left(V_{j, \ell-1}\right)\right)\right]\right) .
\end{aligned}
$$

and

$$
\begin{gathered}
R_{n, 1,1}:=\sum_{\ell \geq 1} Y_{n, \ell} ; \quad Y_{n, \ell}:=\sum_{j=(4 \ell+2)\left\lfloor\frac{n}{4 \ell+2}\right\rfloor+1}^{n} \sum_{i=(4 \ell+2)\left\lfloor\frac{j-1}{4 \ell+2}\right\rfloor+1}^{j-1} H_{i, j}^{(\ell)} \\
R_{n, 1,2}:=\sum_{\ell \geq 1} Z_{n, \ell} ; \quad Z_{n, \ell}:=\sum_{a=1}^{4 \ell+2} \sum_{j=(4 \ell+2)\left\lfloor\frac{n}{4 \ell+2}\right\rfloor+1}^{n} \sum_{k=0}^{\left\lfloor\frac{j-1}{4 \ell+2}\right\rfloor-1} H_{(4 \ell+2) k+a, j}^{(\ell)} .
\end{gathered}
$$

For a fix $\ell \geq 1$, we evaluate the contribution of of $Y_{n, \ell}$ and $Z_{n, \ell}$.

Lemma 2.7. Let $\ell \geq 1$. The following inequalities hold.

$$
\begin{gathered}
\left\|\sup _{n \geq 1} \frac{1}{n^{3 / 2} \sqrt{\mathrm{LL}(n)}}\left|Y_{n, \ell}\right|\right\|_{p, \infty} \leq c_{p} \ell^{1-1 / p} \theta_{\ell, p} \\
\left\|\sup _{n \geq 1} \frac{1}{n^{3 / 2} \sqrt{\mathrm{LL}(n)}}\left|Z_{n, \ell}\right|\right\|_{p, \infty} \leq c_{p} \ell^{2} \theta_{\ell, p} .
\end{gathered}
$$

Proof: First observe that $Y_{n, \ell}$ is a sum of at most $(4 \ell+2)^{2}$ random variables whose weak- $\mathbb{L}^{p}$-norm does not exceed $\theta_{\ell, p}$ hence by cutting the supremum where $n$ is between two consecutive multiples of $4 \ell+2$ gives

$$
\begin{aligned}
\left\|\sup _{n \geq 1} \frac{1}{n^{3 / 2} \sqrt{\mathrm{LL}(n)}}\left|Y_{n, \ell}\right|\right\|_{p, \infty} & \leq\left(\sum_{n \geq 1}\left\|\frac{1}{n^{3 / 2} \sqrt{\mathrm{LL}(n)}}\left|Y_{n, \ell}\right|\right\|_{p, \infty}^{p}\right)^{1 / p} \\
& \leq c(4 \ell+2)^{1-1 / p}\left(\sum_{n \geq 1} n^{-3 p / 2}\right)^{1 / p} \theta_{\ell, p} .
\end{aligned}
$$

In order to treat $Z_{n, \ell}$, we decompose it as $Z_{n, \ell}^{\prime}+Z_{n, \ell}^{\prime \prime}$, where

$$
\begin{gathered}
Z_{n, \ell}^{\prime}=\sum_{a=1}^{4 \ell+2} \sum_{j=(4 \ell+2)\left\lfloor\frac{n}{4 \ell+2}\right\rfloor+1}^{n} \sum_{k=0}^{\left\lfloor\frac{j-1}{4 \ell+2}\right\rfloor-1}\left(H_{(4 \ell+2) k+a, j}^{(\ell)}-\mathbb{E}\left[H_{(4 \ell+2) k+a, j}^{(\ell)} \mid V_{j, \ell}\right]\right) \\
Z_{n, \ell}^{\prime \prime}=\sum_{a=1}^{4 \ell+2} \sum_{j=(4 \ell+2)\left\lfloor\frac{n}{4 \ell+2}\right\rfloor+1}^{n} \sum_{k=0}^{\left\lfloor\frac{j-1}{4 \ell+2}\right\rfloor-1} \mathbb{E}\left[H_{(4 \ell+2) k+a, j}^{(\ell)} \mid V_{j, \ell}\right] .
\end{gathered}
$$


We first use (1.25) to get

$$
\begin{aligned}
\left\|\sup _{n \geq 1} \frac{1}{n^{3 / 2} \sqrt{\mathrm{LL}(n)}}\left|Z_{n, \ell}^{\prime}\right|\right\|_{p, \infty} \leq\left(\sum_{n \geq 3}\left\|\frac{1}{n^{3 / 2} \sqrt{\mathrm{LL}(n)}}\left|Z_{n, \ell}^{\prime}\right|\right\|_{p, \infty}^{p}\right)^{1 / p} \\
\leq\left(\sum_{N \geq 1} N^{-3 p / 2}(4 \ell+2)^{-3 p / 2}\left(\sum_{n=(4 \ell+2) N}^{(4 \ell+2)(N+1)-1}\left\|Z_{n, \ell}^{\prime}\right\|_{p, \infty}\right)^{p}\right)^{1 / p}
\end{aligned}
$$

and for all $n$ such that $(4 \ell+2) N \leq n \leq(4 \ell+2)(N+1)-1$,

$$
\begin{aligned}
& \left\|Z_{n, \ell}^{\prime}\right\|_{p, \infty} \\
& \leq \sum_{a=1}^{4 \ell+2} \sum_{j=(4 \ell+2)\left\lfloor\frac{n}{4 \ell+2}\right\rfloor+1}^{n}\left\|\sum_{k=0}^{\left\lfloor\frac{n}{4 \ell+2}\right\rfloor-1}\left(H_{(4 \ell+2) k+a, j}^{(\ell)}-\mathbb{E}\left[H_{(4 \ell+2) k+a, j}^{(\ell)} \mid V_{j, \ell}\right]\right)\right\|_{p, \infty} \\
& \leq \sum_{a=1}^{4 \ell+2} \sum_{j=(4 \ell+2) N+1}^{(4 \ell+2)(N+1)}\left\|\sum_{k=0}^{N-1}\left(H_{(4 \ell+2) k+a, j}^{(\ell)}-\mathbb{E}\left[H_{(4 \ell+2) k+a, j}^{(\ell)} \mid V_{j, \ell}\right]\right)\right\|_{p, \infty}
\end{aligned}
$$

hence

$$
\begin{aligned}
& \left\|\sup _{n \geq 1} \frac{1}{n^{3 / 2} \sqrt{\mathrm{LL}(n)}}\left|Z_{n, \ell}^{\prime}\right|\right\|_{p, \infty}^{p} \\
& \leq \sum_{N \geq 1} N^{-p-1}(4 \ell+2)^{-3 p / 2} \times \\
& \left(\sum_{a=1}^{4 \ell+2} \sum_{j=(4 \ell+2) N+1}^{(4 \ell+2)(N+1)}\left\|\sum_{k=0}^{N-1}\left(H_{(4 \ell+2) k+a, j}^{(\ell)}-\mathbb{E}\left[H_{(4 \ell+2) k+a, j}^{(\ell)} \mid V_{j, \ell}\right]\right)\right\|_{p, \infty}\right)^{p} .
\end{aligned}
$$

For all fixed $j$, we notice using Lemma A.5 that

$$
\left(H_{(4 \ell+2) k+a, j}^{(\ell)}-\mathbb{E}\left[H_{(4 \ell+2) k+a, j}^{(\ell)} \mid V_{j, \ell}\right]\right)_{0 \leq k \leq N-1}
$$

is a martingale differences sequence with respect to the filtration $\left(\mathcal{F}_{k}\right)_{0 \leq k \leq N-1}$ where

$$
\mathcal{F}_{k}:=\sigma\left(V_{j, \ell}\right) \vee \sigma\left(V_{(4 \ell+2) i+a, j}, i \leq k\right)
$$

hence

$$
\begin{aligned}
& \left\|\sum_{k=0}^{N-1}\left(H_{(4 \ell+2) k+a, j}^{(\ell)}-\mathbb{E}\left[H_{(4 \ell+2) k+a, j}^{(\ell)} \mid V_{j, \ell}\right]\right)\right\|_{p, \infty}^{p} \\
& \leq\left\|\sum_{k=0}^{N-1}\left(H_{(4 \ell+2) k+a, j}^{(\ell)}-\mathbb{E}\left[H_{(4 \ell+2) k+a, j}^{(\ell)} \mid V_{j, \ell}\right]\right)\right\|_{2}^{p} \\
& \leq\left(\sum_{k=0}^{N-1}\left\|\left(H_{(4 \ell+2) k+a, j}^{(\ell)}-\mathbb{E}\left[H_{(4 \ell+2) k+a, j}^{(\ell)} \mid V_{j, \ell}\right]\right)\right\|_{2}^{2}\right)^{p / 2} \leq c_{p} N^{p / 2} \theta_{\ell, 2}^{p}
\end{aligned}
$$


and plugging this estimate into (2.75) gives

$$
\begin{aligned}
& \left\|\sup _{n \geq 1} \frac{1}{n^{3 / 2} \sqrt{\mathrm{LL}(n)}}\left|Z_{n, \ell}^{\prime}\right|\right\|_{p, \infty} \\
& \leq C_{p}\left(\sum_{N \geq 1} N^{-1-p / 2}(4 \ell+2)^{-3 p / 2+2}(4 \ell+2)^{2 p} N \theta_{\ell, p}^{p}\right)^{1 / p} \leq C_{p}^{\prime} \theta_{\ell, 2} \ell^{1 / 2} .
\end{aligned}
$$

We control the contribution of $Z_{n, \ell}^{\prime \prime}$ by noticing that $\mathbb{E}\left[H_{(4 \ell+2) k+a, j}^{(\ell)} \mid V_{j, \ell}\right]$ is actually independent on $k$.

This ends the proof of Lemma 2.7.

2.3.2. Treatment of terms of the form $\sum_{u} H_{a,(4 \ell+2) u+b}^{(\ell)}$ and $\sum_{u} H_{(4 \ell+2) u+a,(4 \ell+2)\left\lfloor\frac{n}{4 \ell+2}\right\rfloor+b}^{(\ell)}$.

Lemma 2.8. For all $\ell \geq 1$ and all $a, b \in[4 \ell+2]$, the following inequality holds

$$
\left\|\sup _{n \geq 1} \frac{1}{n^{3 / 2} \sqrt{\operatorname{LL}(n)}} \mid \sum_{u=1}^{\left\lfloor\frac{n}{4 \ell+2}\right\rfloor} H_{a,(4 \ell+2) u+b}^{(\ell)}\right\| \|_{p, \infty} \leq c_{p}(4 \ell+2)^{-3 / 2} \theta_{\ell, p} .
$$

Lemma 2.9. For all $\ell \geq 1$ and all $a, b \in[4 \ell+2]$, the following inequality holds

$$
\left\|\sup _{n \geq 1} \frac{1}{n^{3 / 2} \sqrt{\operatorname{LL}(n)}} \mid \sum_{u=1}^{\left\lfloor\frac{n}{4 \ell+2}\right\rfloor} H_{(4 \ell+2) u+a,(4 \ell+2)\left\lfloor\frac{n}{4 \ell+2}\right\rfloor+b}^{(\ell)}\right\| \|_{p, \infty} \leq c_{p}(4 \ell+2)^{-1-1 / p} \theta_{\ell, p} .
$$

The proof follows exactly the same idea as the proof of Lemmas 2.4 and 2.5, where the use of Corollary 1.3 is replaced by that of Proposition A.4.

\subsubsection{Treatment of terms of the form $\sum_{u} H_{(4 \ell+2) u+a,(4 \ell+2) u+b}^{(\ell)}$.}

Lemma 2.10. For all $\ell \geq 1$ and all $a, b \in[4 \ell+2]$, the following inequality holds

$$
\left\|\sup _{n \geq 1} \frac{1}{n^{3 / 2}}\left|\sum_{u=1}^{\left\lfloor\frac{n}{4 \ell+2}\right\rfloor} H_{(4 \ell+2) u+a,(4 \ell+2) u+b}^{(\ell)}\right|\right\|_{p, \infty} \leq c_{p}(4 \ell+2)^{-3 / 2} \theta_{\ell, p} .
$$

This follows from the fact that $\left(H_{(4 \ell+2) u+a,(4 \ell+2) u+b}^{(\ell)}\right)_{u \geq 1}$ forms a two-dependent sequence.

2.4. Proof of Theorem 1.\%. Let us explain the idea of the proof. The convergence is essentially due to the partial sums of strictly stationnary sequence $\left(Y_{k}\right)_{k \geq 1}$, where

$$
Y_{k}=Y_{k, 0}+\sum_{\ell \geq 1} Y_{k, \ell}-Y_{k, \ell-1}
$$

and

$$
Y_{k, \ell}:=\mathbb{E}\left[h\left(f_{\ell}\left(V_{k, \ell}\right), f_{\ell}\left(V_{0, \ell}^{\prime}\right)\right) \mid V_{k, \ell}\right]-\mathbb{E}\left[h\left(f_{\ell}\left(V_{k, \ell}\right), f_{\ell}\left(V_{0, \ell}^{\prime}\right)\right)\right] .
$$


We can establish the convergence of $\left(n^{-1 / 2} \sum_{k=1}^{n} Y_{k}\right)_{n \geq 1}$ by showing the convergence of $\left(n^{-1 / 2} \sum_{k=1}^{n} Y_{k, L}\right)_{n \geq 1}$ for a fixed $L$ and by controlling the remainder. We have to prove that all the terms in the decomposition obtained in Proposition 1.1 converge to zero in probability. To sum up, we start by writing

$$
\begin{aligned}
& \frac{1}{n^{3 / 2}} U_{n}\left(h, f,\left(\varepsilon_{i}\right)_{i \in \mathbb{Z}}\right)-\mathbb{E}\left[U_{n}\left(h, f,\left(\varepsilon_{i}\right)_{i \in \mathbb{Z}}\right)\right]=\frac{1}{\sqrt{n}} \sum_{k=1}^{n} Y_{k}+U_{n}^{\text {ind }}\left(h^{(0)},\left(\varepsilon_{i}\right)_{i}\right)+ \\
& \quad+\frac{1}{n^{3 / 2}} \sum_{\ell \geq 1} \sum_{a, b \in[4 \ell+2]} U_{\left\lfloor\frac{n}{2 \ell}\right\rfloor}^{\text {ind }}\left(h_{a, b}^{(\ell)},\left(\varepsilon_{i}^{a, b}\right)\right)+R_{n, 1,1}+R_{n, 1,2}+\sum_{k=2}^{7} R_{n, k}, \quad(2.81)
\end{aligned}
$$

where for each $\ell \geq 1$ and all $a, b \in[4 \ell+2]$, the $U$-statistic $U_{\left\lfloor\frac{n}{2 \ell}\right\rfloor}^{\text {ind }}\left(h_{a, b}^{(\ell)},\left(\varepsilon_{i}^{a, b}\right)\right)$ has independent data and is degenerated, and the remainder terms are defined as

$$
\begin{aligned}
& R_{n, 1,1}:=\frac{1}{n^{3 / 2}} \sum_{\ell \geq 1} \sum_{j=(4 \ell+2)\left\lfloor\frac{n}{4 \ell+2}\right\rfloor+1}^{n} \sum_{i=(4 \ell+2)\left\lfloor\frac{j-1}{4 \ell+2}\right\rfloor+1}^{j-1} H_{i, j}^{(\ell)} \\
& R_{n, 1,2}:=\frac{1}{n^{3 / 2}} \sum_{\ell \geq 1} \sum_{a=1}^{4 \ell+2} \sum_{j=(4 \ell+2)\left\lfloor\frac{n}{4 \ell+2}\right\rfloor+1}^{n} \sum_{k=0}^{\left\lfloor\frac{j-1}{4 \ell+2}\right\rfloor-1} H_{(4 \ell+2) k+a, j}^{(\ell)}
\end{aligned}
$$

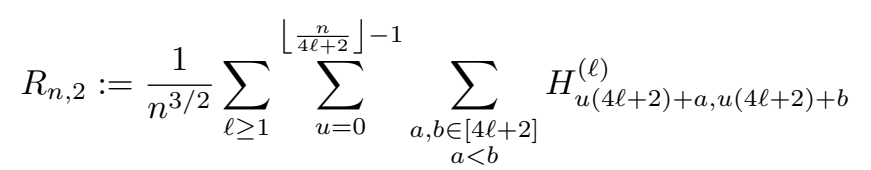

$$
\begin{aligned}
& R_{n, 3}:=\frac{1}{n^{3 / 2}} \sum_{\ell \geq 1} \sum_{v=1}^{\left\lfloor\frac{n}{4 \ell+2}\right\rfloor-1} \sum_{\substack{a, b \in[4 \ell+2] \\
0 \leq a-b \leq(2 \ell+1)-1}}\left(H_{a, v(4 \ell+2)+b}^{(\ell)}+H_{b, v(4 \ell+2)+a}^{(\ell)}\right)
\end{aligned}
$$

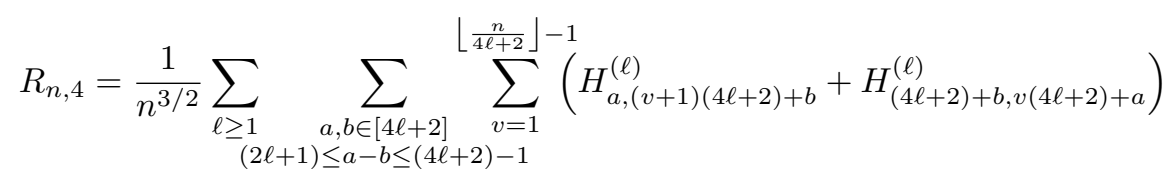

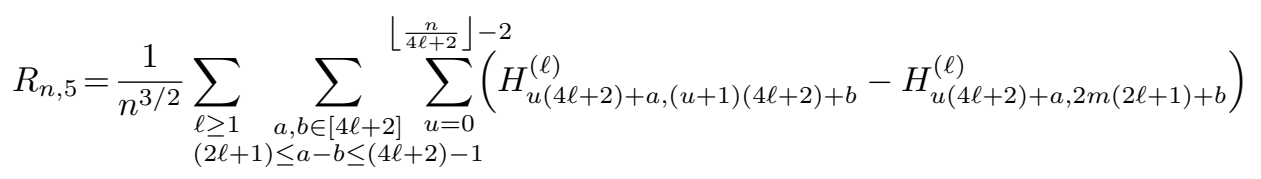

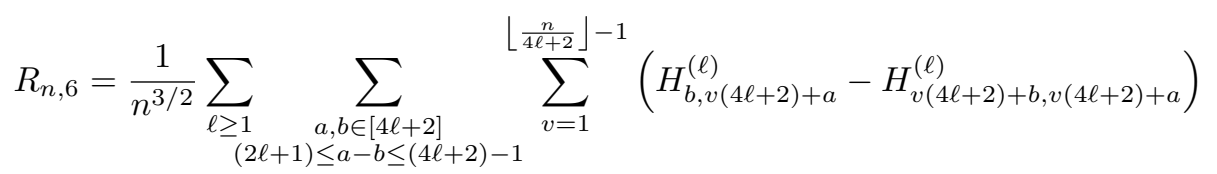

$$
\begin{aligned}
& R_{n, 7}:=\frac{1}{n^{3 / 2}} \sum_{\ell \geq 1} R_{n, 7}^{(\ell)}, \\
& R_{n, 7}^{(\ell)}:=(4 \ell+1)\left\lfloor\frac{n}{4 \ell+1}\right\rfloor \sum_{k=1}^{(4 \ell+2)\left\lfloor\frac{n}{4 \ell+2}\right\rfloor+1}\left(Y_{k, \ell}-Y_{k, \ell-1}\right)-n \sum_{k=1}^{n}\left(Y_{k, \ell}-Y_{k, \ell-1}\right)
\end{aligned}
$$


with

$$
\begin{aligned}
H_{i, j}^{(\ell)}:= & h\left(f_{\ell}\left(V_{i, \ell}\right), f_{\ell}\left(V_{j, \ell}\right)\right)-\mathbb{E}\left[h\left(f_{\ell}\left(V_{i, \ell}\right), f_{\ell}\left(V_{j, \ell}\right)\right)\right] \\
& -\left(h\left(f_{\ell-1}\left(V_{i, \ell-1}\right), f_{\ell-1}\left(V_{j, \ell-1}\right)\right)-\mathbb{E}\left[h\left(f_{\ell-1}\left(V_{i, \ell-1}\right), f_{\ell-1}\left(V_{j, \ell-1}\right)\right)\right]\right) .
\end{aligned}
$$

We then follow the steps:

(1) we show that $\left(n^{-1 / 2} \sum_{k=1}^{n} Y_{k}\right)_{n \geq 1}$ converges to a centered normal distribution with variance

(2) We show the convergence in probability to zero of all the terms $R_{n, 1,1}$, $R_{n, 1,2}, R_{n, k}, 2 \leq k \leq 7$.

2.4.1. Convergence of $\left(n^{-1 / 2} \sum_{k=1}^{n} Y_{k}\right)_{n \geq 1}$. We use Theorem 4.2 in Billingsley (1968), which states the following. For $L, n \in \mathbb{N}, Z_{n}, Z_{n, L}, W_{L}$ and $Z$ are real-valued random variables defined on a common probability space $(\Omega, \mathcal{F}, \mathbb{P})$. We assume that

(1) for all $L \in \mathbb{N}, Z_{n, L} \rightarrow W_{L}$ in distribution as $n \rightarrow \infty$;

(2) $W_{L} \rightarrow Z$ in distribution as $L \rightarrow \infty$, and

(3) for each $\varepsilon>0, \lim _{L \rightarrow \infty} \lim \sup _{n \rightarrow \infty} \mathbb{P}\left\{\left|Z_{n, L}-Z_{n}\right|>\varepsilon\right\}=0$.

Then $Z_{n} \rightarrow Z$ in distribution as $n \rightarrow \infty$.

We will apply the result in the following setting:

$$
Z_{n, L}:=\frac{1}{\sqrt{n}} \sum_{k=1}^{n} Y_{k, L}
$$

$W_{L}$ a centered normal variable with variance $\sigma_{L}^{2}:=\sum_{k=-L}^{L} \operatorname{Cov}\left(Y_{0, L}, Y_{k, L}\right)$ and $W$ a centered normal variable with variance $\sigma^{2}=\sum_{k \in \mathbb{Z}} \operatorname{Cov}\left(Y_{0}, Y_{k}\right)$.

The first item follows from the central limit theorem for $(2 L+1)$-dependent random variables; the second one from the convergence of $\left(\sigma_{L}^{2}\right)_{L \geq 1}$ to $\sigma^{2}$, which can be seen by writing $Y_{k, L}-Y_{k}=\sum_{\ell \geq L}\left(Y_{k, \ell}-Y_{k, \ell+1}\right)$ and using Cauchy-Schwarz inequality:

$$
\begin{aligned}
&\left|\sum_{k=-L}^{L} \operatorname{Cov}\left(Y_{0, L}, Y_{k, L}\right)-\operatorname{Cov}\left(Y_{0}, Y_{k}\right)\right| \\
& \leq \sum_{k=-L}^{L}\left|\operatorname{Cov}\left(Y_{0, L}, Y_{k, L}-Y_{k}\right)\right|+\sum_{k=-L}^{L}\left|\operatorname{Cov}\left(Y_{0, L}-Y_{0}, Y_{k}\right)\right| \\
& \leq 2 L\left\|Y_{0, L}\right\|_{2} \sum_{\ell \geq L} \theta_{\ell, 2}+2 L\left\|Y_{k}\right\|_{2} \sum_{\ell \geq L} \theta_{\ell, 2}
\end{aligned}
$$

and the quantities $\left\|Y_{0, L}\right\|_{2}$ and $\left\|Y_{k}\right\|_{2}$ are bounded independently of $L$ and $k$.

For the third item, we start from Chebytchev's inequality:

$$
\mathbb{P}\left\{\left|Z_{n, L}-Z_{n}\right|>\varepsilon\right\} \leq \varepsilon^{-2}\left\|Z_{n, L}-Z_{n}\right\|_{2}^{2}
$$

and

$$
\left\|Z_{n, L}-Z_{n}\right\|_{2} \leq \sum_{\ell \geq L}\left\|\frac{1}{\sqrt{n}} \sum_{k=1}^{n}\left(Y_{k, \ell}-Y_{k, \ell-1}\right)\right\|_{2}
$$


and $\left\|\frac{1}{\sqrt{n}} \sum_{k=1}^{n}\left(Y_{k, \ell}-Y_{k, \ell-1}\right)\right\|_{2}$ does not exceed a constant times $\ell^{1 / 2} \theta_{\ell, 2}$ hence

$$
\limsup _{n \rightarrow+\infty} \mathbb{P}\left\{\left|Z_{n, L}-Z_{n}\right|>\varepsilon\right\} \leq \varepsilon^{-2}\left(\sum_{\ell \geq L} \ell^{1 / 2} \theta_{\ell, 2}\right)^{2} .
$$

2.4.2. Convergence in probability of $R_{n, 1,1}, R_{n, 1,2}$ and $R_{n, k}, 2 \leq k \leq 6$ to 0 . Observe that

$$
\mathbb{E}\left[\left|R_{n, 1,1}\right|\right] \leq \frac{1}{n^{3 / 2}} \sum_{\ell \geq 1} \sum_{j=(4 \ell+2)\left\lfloor\frac{n}{4 \ell+2}\right\rfloor+1}^{n} \sum_{i=(4 \ell+2)\left\lfloor\frac{j-1}{4 \ell+2}\right\rfloor+1}^{j-1}\left\|H_{i, j}^{(\ell)}\right\|_{1},
$$

that $\left\|H_{i, j}^{(\ell)}\right\|_{1} \leq \theta_{\ell, 1}$ and that the number of terms in the two inner sums is of order $\ell^{2}$ hence

$$
\mathbb{E}\left[\left|R_{n, 1,1}\right|\right] \leq \frac{1}{n^{3 / 2}} \sum_{\ell \geq 1} \ell^{2} \theta_{\ell, 1} .
$$

We use the same method for the terms $R_{n, 1,2}$ and $R_{n, k}, 2 \leq k \leq 6$.

2.4.3. Treatment of $R_{n, 7}$. First we rewrite $R_{n, 7}$ as a double sum, namely, as

$$
R_{n, 7}=\frac{1}{n^{3 / 2}} \sum_{\ell \geq 1} \sum_{k \geq 1}\left(Y_{k, \ell}-Y_{k, \ell-1}\right) c_{n, k, \ell}
$$

where

$$
c_{n, k, \ell}=(4 \ell+2)\left\lfloor\frac{n}{4 \ell+2}\right\rfloor\left[k \leq(4 \ell+2)\left\lfloor\frac{n}{4 \ell+2}\right\rfloor+1\right]-n[k \leq n],
$$

with the notation $[P]=1$ if the assertion $P$ holds and 0 otherwise. Write $n$ as $(4 \ell+2) N+r$, where $0 \leq r \leq 4 \ell+1$. Then

$$
c_{n, k, \ell}=-q[k \leq N(4 \ell+2)+1]-n[N(4 \ell+2) \leq k \leq n],
$$

hence

$$
\begin{aligned}
\left|R_{n, 7}\right| \leq \frac{1}{n^{3 / 2}} \sum_{\ell \geq 1} \ell\left|\sum_{k=1}^{(4 \ell+2)\left\lfloor\frac{n}{4 \ell+2}\right\rfloor+1}\left(Y_{k, \ell}-Y_{k, \ell-1}\right)\right| & \\
& +\frac{1}{n^{1 / 2}} \sum_{\ell \geq 1}\left|\sum_{(4 \ell+2)\left\lfloor\frac{n}{4 \ell+2}\right\rfloor+1}^{n}\left(Y_{k, \ell}-Y_{k, \ell-1}\right)\right|
\end{aligned}
$$

and taking the expectation gives that

$$
\left\|R_{n, 7}\right\|_{1} \leq 2 n^{-1 / 2} \sum_{\ell \geq 1} \ell \theta_{\ell, 1}
$$


2.5. Proof of Theorem 1.13. We start the proof of Theorem 1.13 by given a bound on the dependence coefficients in terms of the $\mathbb{L}^{r}$-norm of the density of the random variable $\mathbb{E}\left[X_{0} \mid \sigma\left(\varepsilon_{u},-\ell \leq u \leq \ell\right)\right]-\mathbb{E}\left[X_{j} \mid \sigma\left(\varepsilon_{u}, j-\ell \leq u \leq j+\ell\right)\right]$ and the $\mathbb{L}^{q_{-}}$ norm of $\mathbb{E}\left[X_{0} \mid V_{0, \ell}\right]-\mathbb{E}\left[X_{0} \mid V_{0, \ell-1}\right]$ :

$$
\theta_{\ell, p} \leq C\left\|\pi_{\ell}\right\|_{q}^{\frac{q}{p} \frac{r}{q(r-1)+r}},
$$

where $C$ is independent of $\ell$.

Let us show (2.93). By the definition of the dependence coefficients (1.8), we have to control $\sup _{j \geq 0}\left\|h\left(f_{\ell}\left(V_{0, \ell}\right), f_{\ell}\left(V_{j, \ell}\right)\right)-h\left(f_{\ell-1}\left(V_{0, \ell-1}\right), f_{\ell-1}\left(V_{j, \ell-1}\right)\right)\right\|_{p}$ with $h(x, y):=\mathbf{1}\{|x-y| \leq t\}$. We will control for a fixed $\ell$ and $j$ the quantity $\left\|h\left(f_{\ell}\left(V_{0, \ell}\right), f_{\ell}\left(V_{j, \ell}\right)\right)-h\left(f_{\ell-1}\left(V_{0, \ell-1}\right), f_{\ell-1}\left(V_{j, \ell-1}\right)\right)\right\|_{p}$. The last quantity involves four random variables, that we will define as

$$
\begin{aligned}
& X:=\mathbb{E}\left[X_{0} \mid \sigma\left(\varepsilon_{u},-\ell \leq u \leq \ell\right)\right], \\
& X^{\prime}:=\mathbb{E}\left[X_{0} \mid \sigma\left(\varepsilon_{u},-\ell+1 \leq u \leq \ell-1\right)\right] \\
& Y:=\mathbb{E}\left[X_{j} \mid \sigma\left(\varepsilon_{u}, j-\ell \leq u \leq j+\ell\right)\right] \\
& Y^{\prime}:=\mathbb{E}\left[X_{j} \mid \sigma\left(\varepsilon_{u}, j-\ell+1 \leq u \leq j+\ell-1\right)\right]
\end{aligned}
$$

in order to ease the notations. For each fixed $\delta$, the following inequalities take place:

$$
\begin{aligned}
& \left\|h\left(f_{\ell}\left(V_{0, \ell}\right), f_{\ell}\left(V_{j, \ell}\right)\right)-h\left(f_{\ell-1}\left(V_{0, \ell-1}\right), f_{\ell-1}\left(V_{j, \ell-1}\right)\right)\right\|_{p} \\
= & \left\|\mathbf{1}\{|X-Y| \leq t\}-\mathbf{1}\left\{\left|X^{\prime}-Y^{\prime}\right| \leq t\right\}\right\|_{p} \\
\leq & \left\|\mathbf{1}\{|X-Y| \leq t\}-\mathbf{1}\left\{\left|X^{\prime}-Y\right| \leq t\right\}\right\|_{p} \\
& +\left\|\mathbf{1}\left\{\left|X^{\prime}-Y\right| \leq t\right\}-\mathbf{1}\left\{\left|X^{\prime}-Y^{\prime}\right| \leq t\right\}\right\|_{p} \\
\leq & \mathbb{P}\left\{\left|X-X^{\prime}\right|>\delta\right\}^{1 / p} \\
& +\left\|\left(\mathbf{1}\{|X-Y| \leq t\}-\mathbf{1}\left\{\left|X^{\prime}-Y\right| \leq t\right\}\right) \mathbf{1}\left\{\left|X-X^{\prime}\right| \leq \delta\right\}\right\|_{p} \\
& +\mathbb{P}\left\{\left|Y-Y^{\prime}\right|>\delta\right\}^{1 / p} \\
& +\left\|\left(\mathbf{1}\left\{\left|X^{\prime}-Y\right| \leq t\right\}-\mathbf{1}\left\{\left|X^{\prime}-Y^{\prime}\right| \leq t\right\}\right) \mathbf{1}\left\{\left|Y-Y^{\prime}\right| \leq \delta\right\}\right\|_{p} .
\end{aligned}
$$

The terms $\mathbb{P}\left\{\left|X-X^{\prime}\right|>\delta\right\}^{1 / p}$ and $\mathbb{P}\left\{\left|Y-Y^{\prime}\right|>\delta\right\}^{1 / p}$ can be bounded by Markov's inequality, giving

$$
\begin{aligned}
& \mathbb{P}\left\{\left|X-X^{\prime}\right|>\delta\right\}^{1 / p}+\mathbb{P}\left\{\left|Y-Y^{\prime}\right|>\delta\right\}^{1 / p} \leq \\
& \delta^{-q / p}\left\|X-X^{\prime}\right\|_{q}^{q / p}+\delta^{-q / p}\left\|Y-Y^{\prime}\right\|_{q}^{q / p}=2 \delta^{-q / p}\left\|X-X^{\prime}\right\|_{q}^{q / p},
\end{aligned}
$$

where we used in the last equality the fact that $X-X^{\prime}$ has the same distribution as $Y-Y^{\prime}$. Moreover, observe that $\mathbf{1}\{|X-Y| \leq t\}-\mathbf{1}\left\{\left|X^{\prime}-Y\right| \leq t\right\}$ is equal to one if $|X-Y| \leq t$ and $\left|X^{\prime}-Y\right|>t$ or the other way around. In both cases, the additional information that $\left|X-X^{\prime}\right| \leq \delta$ shows that $|X-Y|$ belongs to the interval $[t-\delta, t+\delta]$. Using a similar argument for the control of $\left\|\left(\mathbf{1}\left\{\left|X^{\prime}-Y\right| \leq t\right\}-\mathbf{1}\left\{\left|X^{\prime}-Y^{\prime}\right| \leq t\right\}\right) \mathbf{1}\left\{\left|Y-Y^{\prime}\right| \leq \delta\right\}\right\|_{p}$, we derive the bound

$$
\begin{aligned}
\| h\left(f_{\ell}\left(V_{0, \ell}\right), f_{\ell}\left(V_{j, \ell}\right)\right)-h\left(f_{\ell-1}\left(V_{0, \ell-1}\right),\right. & \left.f_{\ell-1}\left(V_{j, \ell-1}\right)\right) \|_{p} \\
\leq 2 \delta^{-q / p}\left\|X-X^{\prime}\right\|_{q}^{q / p}+\mathbb{P}\{t- & \delta \leq|X-Y| \leq t+\delta\}^{1 / p} \\
& +\mathbb{P}\left\{t-\delta \leq\left|X^{\prime}-Y^{\prime}\right| \leq t+\delta\right\}^{1 / p} .
\end{aligned}
$$


Now, using the assumption on the density of $X-Y$ and $X^{\prime}-Y^{\prime}$, we obtain

$$
\begin{aligned}
\| h\left(f_{\ell}\left(V_{0, \ell}\right), f_{\ell}\left(V_{j, \ell}\right)\right)-h\left(f_{\ell-1}(\right. & \left.\left.V_{0, \ell-1}\right), f_{\ell-1}\left(V_{j, \ell-1}\right)\right) \|_{p} \\
& \leq 2 \delta^{-q / p}\left\|X-X^{\prime}\right\|_{q}^{q / p}+4\left\|g_{k, \ell}\right\|_{\mathbb{L}^{q}(\mathbb{R})} \delta^{\frac{r}{p(r-1)}} .
\end{aligned}
$$

We choose $\delta$ in such a way that the last two terms have a similar contribution equal. This leads to the choice $\delta=\left\|X-X^{\prime}\right\|_{q}^{\frac{q}{q+\frac{r}{r-1}}}$, from which inequality

$$
\begin{aligned}
& \left\|h\left(f_{\ell}\left(V_{0, \ell}\right), f_{\ell}\left(V_{j, \ell}\right)\right)-h\left(f_{\ell-1}\left(V_{0, \ell-1}\right), f_{\ell-1}\left(V_{j, \ell-1}\right)\right)\right\|_{p} \\
\leq & C\left\|\mathbb{E}\left[X_{0} \mid \sigma\left(\varepsilon_{u},-\ell \leq u \leq \ell\right)\right]-\mathbb{E}\left[X_{0} \mid \sigma\left(\varepsilon_{u},-\ell+1 \leq u \leq \ell-1\right)\right]\right\|_{q}^{\frac{q}{p} \frac{r}{q(r-1)+r}}
\end{aligned}
$$

follows, giving (2.93).

Now, the law of large numbers and the bounded law of the iterated logarithms follow from the combination of (2.93) with Theorem 1.5 and Theorem 1.4 respectively. For the central limit theorem, we use again (2.93) in order to check that (1.33) holds. It remains to verify (1.34) which will be done by controlling each term of the series in (1.40). To this aim, let $Z_{k}$ and $Z_{k}^{\prime}$ be the random variables involved in the statement. We have to show that

$$
\sum_{k \in \mathbb{Z}}\left\|\mathbf{1}\left\{\left|Z_{k}-X_{0}^{\prime}\right| \leq t\right\}-\mathbf{1}\left\{\left|Z_{k}^{\prime}-X_{0}^{\prime}\right| \leq t\right\}\right\|_{2},
$$

where $X_{0}^{\prime}=f\left(\left(\varepsilon_{-u}^{\prime}\right)_{u \in \mathbb{Z}}\right)$. Use use the same method as before in order to control the quantity $\left\|\mathbf{1}\left\{\left|Z_{k}-X_{0}^{\prime}\right| \leq t\right\}-\mathbf{1}\left\{\left|Z_{k}^{\prime}-X_{0}^{\prime}\right| \leq t\right\}\right\|_{2}$ for each fixed $k$, that is, we intersect over $\left\{\left|Z_{k}-Z_{k}^{\prime}\right| \leq \delta\right\}$ for some well chosen $\delta$, namely, $\delta=$ $\left\|Z_{k}-Z_{k}^{\prime}\right\|_{q}^{\frac{q(r-1)}{r+q(r-1)}}$. This ends the proof of Theorem 1.13.

\section{A. Appendix}

In this appendix, we collect some fact about partial sums of martingales or functional of independent sequences that we will need in the proof.

The first is a probability inequality for martingales; Proposition A.2 and A.3 give a control of the maximal function involved in the strong law of large numbers, respectively for martingales and functionals of a fixed number of i.i.d. random variables. We end the Appendix by two lemmas on conditional expectation.

Proposition A.1 (Theorem 1.3 in Giraudo, 2019). Let $1<p<2$ and $q>p$. Then there exists constants $c_{1}$ and $c_{2}$ depending only on $\{p, q\}$ such that if $\left(d_{i}\right)_{i=1}^{n}$ is a martingale differences sequence with respect to a filtration $\left(\mathcal{F}_{i}\right)_{i=1}^{n}$, then for each integer $n$ and each positive $x$,

$$
\begin{aligned}
\mathbb{P}\left\{\left|\sum_{i=1}^{n} d_{i}\right|>x\right\} & \leq c_{1} \sum_{i=1}^{n} \int_{0}^{1} \mathbb{P}\left\{\left|d_{i}\right|>x u c_{2}\right\} u^{q-1} \mathrm{~d} u \\
& +c_{1} \int_{0}^{1} \mathbb{P}\left\{\left(\sum_{i=1}^{n} \mathbb{E}\left[\left|d_{i}\right|^{p} \mid \mathcal{F}_{i-1}\right]\right)^{1 / p}>x u c_{2}\right\} u^{q-1} \mathrm{~d} u .
\end{aligned}
$$

The next Proposition gives a control of the maximal function involved in the strong law of large numbers. A control on the $r$-th moment for $r<p$ was obtained 
in the proof of Theorem 4.1 in Woyczyński (1982). The control on the weak- $\mathbb{L}^{p}$ moment was explicitely established in Cuny (2015), p. 324, under a stationarity assumption, but the proof works for martingale with identically distributed increments.

Proposition A.2. Let $\left(d_{j}\right)_{j \geq 1}$ be an identically distributed martingale differences sequence with respect to the filtration $\left(\mathcal{F}_{j}\right)_{j \geq 0}$. Then for all $1<p<2$,

$$
\left\|\sup _{n \geq 1} \frac{1}{n^{1 / p}}\left|\sum_{j=1}^{n} d_{j}\right|\right\|_{p, \infty} \leq c_{p}\left\|d_{1}\right\|_{p} .
$$

Proposition A.3. Let $\ell \geq 1$ and $\left(X_{j}\right)_{j \geq 1}$ be a sequence such that $X_{j}=$ $f\left(\left(\varepsilon_{u}\right)_{u=j-\ell}^{j+\ell}\right)$, where $\left(\varepsilon_{u}\right)_{u \in \mathbb{Z}}$ is i.i.d. and $X_{1}$ is centered. Then for all $1<p<2$,

$$
\left\|\sup _{n \geq 1} \frac{1}{n^{1 / p}}\left|\sum_{j=1}^{n} X_{j}\right|\right\|_{p, \infty} \leq c_{p} \ell^{1-1 / p}\left\|X_{1}\right\|_{p} .
$$

Proof: We first notice that

$$
\sup _{n \geq 1} \frac{1}{n^{1 / p}}\left|\sum_{j=1}^{n} X_{j}\right| \leq \sum_{a \in[4 \ell+2]} \sup _{n \geq 1} \frac{1}{n^{1 / p}}\left|\sum_{k=1}^{\left\lfloor\frac{n}{4 \ell+2}\right\rfloor} X_{(4 \ell+2) k+a}\right|
$$

and we apply Proposition A.2 to the sequences $\left(X_{(4 \ell+2) k+a}\right)_{k \geq 1}$ for all fixed $a \in$ $[4 \ell+2]$.

The next Proposition give a control of the weak- $\mathbb{L}^{p}$-norm of the maximum function involved in the bounded law of the iterated logarithms for partial sum of stationary sequences. When the involved sequence is i.i.d. and centered, this reduces to Théorème 1 in Pisier (1976). This can be extended to the context of the functional of $2 \ell+1$ i.i.d. random variables by the same method as in the proof of Proposition A.4.

Proposition A.4. Let $\ell \geq 1$ and $\left(X_{j}\right)_{j \geq 1}$ be a sequence such that $X_{j}=$ $f\left(\left(\varepsilon_{u}\right)_{u=j-\ell}^{j+\ell}\right)$, where $\left(\varepsilon_{u}\right)_{u \in \mathbb{Z}}$ is i.i.d. and $X_{1}$ is centered. Then for all $1<p<2$,

$$
\left\|\sup _{n \geq 1} \frac{1}{\sqrt{n \mathrm{LL}(n)}}\left|\sum_{j=1}^{n} X_{j}\right|\right\|_{p, \infty} \leq c_{p} \ell^{1 / 2}\left\|X_{1}\right\|_{2} .
$$

Lemma A.5 (Proposition 2 p. 1693 in Wang and Woodroofe, 2013). Let $Y$ be a real-valued random variable and let $\mathcal{F}$ and $\mathcal{G}$ be two sub- $\sigma$-algebra such that $\mathcal{G}$ is independent of the $\sigma$-algebra generated by $Y$ and $\mathcal{F}$. Then

$$
\mathbb{E}[Y \mid \mathcal{F} \vee \mathcal{G}]=\mathbb{E}[Y \mid \mathcal{F}]
$$

Finally, the next lemma is well-known.

Lemma A.6. Let $Y$ and $Z$ be two independent random variables with values in $\mathbb{R}^{d}$. Let $f: \mathbb{R}^{d} \times \mathbb{R}^{d} \rightarrow \mathbb{R}$ be a measurable function. Then

$$
\mathbb{E}[f(Y, Z) \mid \sigma(Z)]=g(Z),
$$

where $g: \mathbb{R}^{d} \rightarrow \mathbb{R}$ is defined by $g(z)=\mathbb{E}[f(Y, z)]$. 


\section{Acknowledgements}

The author is grateful to both referees for many inspiring comments which led to a substantial improvement of the paper.

\section{References}

Aaronson, J., Burton, R., Dehling, H., Gilat, D., Hill, T., and Weiss, B. Strong laws for $L$ - and $U$-statistics. Trans. Amer. Math. Soc., 348 (7), 2845-2866 (1996). MR1363941.

Arcones, M. A. On the central limit theorem for $U$-statistics under absolute regularity. Statist. Probab. Lett., 24 (3), 245-249 (1995). MR1353588.

Arcones, M. A. The law of large numbers for $U$-statistics under absolute regularity. Electron. Comm. Probab., 3, 13-19 (1998a). MR1624866.

Arcones, M. A. The law of the large numbers for $U$-statistics for $2 m$-wise independent random variables. Statist. Probab. Lett., 40 (1), 75-81 (1998b). MR1650528.

Arcones, M. A. and Giné, E. On the law of the iterated logarithm for canonical $U$-statistics and processes. Stochastic Process. Appl., 58 (2), 217-245 (1995). MR1348376.

Beutner, E. and Zähle, H. Deriving the asymptotic distribution of U- and Vstatistics of dependent data using weighted empirical processes. Bernoulli, 18 (3), 803-822 (2012). MR2948902.

Billingsley, P. Convergence of probability measures. John Wiley \& Sons, Inc., New York-London-Sydney (1968). MR0233396.

Borovkova, S., Burton, R., and Dehling, H. Limit theorems for functionals of mixing processes with applications to $U$-statistics and dimension estimation. Trans. Amer. Math. Soc., 353 (11), 4261-4318 (2001). MR1851171.

Cuny, C. A compact LIL for martingales in 2-smooth Banach spaces with applications. Bernoulli, 21 (1), 374-400 (2015). MR3322323.

Dehling, H. and Sharipov, O. S. Marcinkiewicz-Zygmund strong laws for $U$ statistics of weakly dependent observations. Statist. Probab. Lett., 79 (19), 20282036 (2009). MR2571765.

Dehling, H. and Wendler, M. Central limit theorem and the bootstrap for $U$ statistics of strongly mixing data. J. Multivariate Anal., 101 (1), 126-137 (2010a). MR2557623.

Dehling, H. and Wendler, M. Law of the iterated logarithm for $U$-statistics of weakly dependent observations. In Dependence in probability, analysis and number theory, pp. 177-194. Kendrick Press, Heber City, UT (2010b). MR2731072.

Denker, M. and Keller, G. Rigorous statistical procedures for data from dynamical systems. J. Statist. Phys., 44 (1-2), 67-93 (1986). MR854400.

Dewan, I. and Prakasa Rao, B. L. S. Central limit theorem for $U$-statistics of associated random variables. Statist. Probab. Lett., 57 (1), 9-15 (2002). MR1911807.

Giné, E. and Zinn, J. Marcinkiewicz type laws of large numbers and convergence of moments for $U$-statistics. In Probability in Banach spaces, 8 (Brunswick, ME, 1991), volume 30 of Progr. Probab., pp. 273-291. Birkhäuser Boston, Boston, MA (1992). MR1227625.

Giraudo, D. Deviation inequalities for Banach space valued martingales differences sequences and random fields. ESAIM Probab. Stat., 23, 922-946 (2019). MR4046858. 
Hoeffding, W. A class of statistics with asymptotically normal distribution. Ann. Math. Statistics, 19, 293-325 (1948). MR26294.

Hsing, T. and $\mathrm{Wu}, \mathrm{W}$. B. On weighted $U$-statistics for stationary processes. Ann. Probab., 32 (2), 1600-1631 (2004). MR2060311.

Leucht, A. Degenerate $U$ - and $V$-statistics under weak dependence: asymptotic theory and bootstrap consistency. Bernoulli, 18 (2), 552-585 (2012). MR2922461.

Pisier, G. Sur la loi du logarithme itéré dans les espaces de Banach. In Probability in Banach spaces (Proc. First Internat. Conf., Oberwolfach, 1975), pp. 203-210. Lecture Notes in Math., Vol. 526 (1976). MR0501237.

Podolskij, M., Schmidt, C., and Ziegel, J. F. Limit theorems for nondegenerate $U$ statistics of continuous semimartingales. Ann. Appl. Probab., 24 (6), 2491-2526 (2014). MR3262509.

Reitzner, M. and Schulte, M. Central limit theorems for $U$-statistics of Poisson point processes. Ann. Probab., 41 (6), 3879-3909 (2013). MR3161465.

Serinko, R. J. A consistent approach to least squares estimation of correlation dimension in weak Bernoulli dynamical systems. Ann. Appl. Probab., 4 (4), 1234-1254 (1994). MR1304784.

Wang, Y. and Woodroofe, M. A new condition for the invariance principle for stationary random fields. Statist. Sinica, 23 (4), 1673-1696 (2013). MR3222815.

Wolff, R. C. L. A note on the behaviour of the correlation integral in the presence of a time series. Biometrika, 77 (4), 689-697 (1990). MR1086682.

Woyczyński, W. A. Asymptotic behavior of martingales in Banach spaces. II. In Martingale theory in harmonic analysis and Banach spaces (Cleveland, Ohio, 1981), volume 939 of Lecture Notes in Math., pp. 216-225. Springer, Berlin-New York (1982). MR668549.

Wu, W. B. Nonlinear system theory: another look at dependence. Proc. Natl. Acad. Sci. USA, 102 (40), 14150-14154 (2005). MR2172215.

$\mathrm{Wu}, \mathrm{W}$. B. Empirical processes of stationary sequences. Statist. Sinica, 18 (1), 313-333 (2008). MR2384990. 